\title{
From molecular machines to stimuli-responsive materials
}

Emilie Moulin, Lara Faour, Christian Camilo Carmona-Vargas, and Nicolas Giuseppone*

Dedicated to Prof. Jean-Pierre Sauvage on the occasion of his 75th Birthday.

Dr. E. Moulin, Dr. L. Faour, C. C. Carmona-Vargas, Prof. Dr. N. Giuseppone

SAMS Research Group, Institut Charles Sadron, CNRS - UPR 22, University of Strasbourg, 23 rue du Loess, BP 84047, 67034 Strasbourg Cedex 2, France

E-mail: giuseppone@unistra.fr

Keywords: molecular machines, molecular motors, mechanical bonds, collective motions, smart materials

Artificial molecular machines are able to produce and to exploit precise nanoscale actuations in response to chemical or physical triggers. Recent scientific efforts have been devoted to the integration, the orientation, and the interfacing of large assemblies of molecular machines in order to harness their collective actuations at larger length scale and up to the generation of macroscopic motions. Making use of such a "hierarchical mechanics" represents a fundamentally new approach for the conception of stimuli-responsive materials. Furthermore, because some molecular machines can function as molecular motors - which are capable of cycling a unidirectional motion out of thermodynamic equilibrium, and of progressively increasing the work delivered to their environment - one can expect unique opportunities to design new kinds of mechanically active materials and devices capable of autonoumous behavior when supplied by an external source of energy. Here, recent achievements of the literature are reported, including the integration of molecular machines at surfaces and interfaces, in 3D self-assembled materials, as well as in liquid crystals and polymer materials. Their detailed functioning principles as well as their functional properties are discussed along with their potential applications in various domains such as sensing, drug delivery, electronics, optics, plasmonics, and mechanics. 


\section{Introduction}

Stimuli-responsive materials are able to sense their environment and to subsequently modify their intrinsic chemical or physical properties. ${ }^{[1,2]}$ These interactive, adaptive, and selfregulating features make them very attractive to operate as smart and multifunctional materials in various domains of research including drug delivery, bio-sensing, tissue engineering, 4D printing, smart coatings, self-healing and recyclable polymers, as well as actuators and artificial muscles. While still at the very beginning of their development, there are now more and more evidences around us that such materials have the potential to strongly impact key technological sectors such as medicine, robotics, information technologies, and environment. A particularly intriguing and large class of artificial stimuli-responsive materials is based on the regulation of their mechanical properties. Shape-memory effects and mechanical actuations can be indeed generated from objects of various chemical nature $\mathrm{e}^{[3-5]}$ (e.g. metallic alloys, ceramics, liquid crystals, or polymers), and they are often supported by a combination of bottom-up and top-down structural engineering techniques going from surfaces to 3D materials (e.g. supramolecular self-assembly, photo-patterning, microfluidics, or inkjet printing). ${ }^{[6,7]}$ Various stimuli can generate their response (e.g. molecules, temperature, light, electrical potential, or mechanical stress) and their functioning principles vary largely from one system to another, involving a number of physical phenomena which can take place at the (macro)molecular level and which are transferred towards higher length scales (e.g. polarity, solubility - such as lower critical solution temperature (LCST), osmotic pressure, surface energy, or phase transition). It should also be mentioned that bio-inspiration is often a driving force in the design of such artificial materials as many smart mechano-active systems with adaptable properties are found in nature (e.g. stiffness change of sea cucumber, adaptive toughness of spider silk, closure of mimosa leaves when touched, or opening of seed pods). ${ }^{[8-}$ 10] 
Since the early 2000s, exciting opportunities have emerged in the scientific community for making use of artificial molecular machines as the elementary responsive building blocks in new types of stimuli-responsive materials. This approach is fundamentally disruptive compared to the others developed so far, because it aims to exploit precisely controlled mechanical motions at molecular level (produced by the machines), and to amplify them in collective mechanical motions taking place at larger dimensions up to the material's length scale. The most prominent example of what could be potentially achieved in the far future by such artificial materials is also found in nature, in the form of striated muscle tissue. In muscles, by using ATP as chemical fuel, millions of myosin motors, which individually cycle hundreds of few nanometers scale actuations, are able to pull synchronously on sliding actin filaments and to produce micrometric contractions of sarcomeric units. Further hierarchical organizations of sarcomeres in bundled fibrils and fibers result in a macroscopic contraction of the muscle. At that point, and before describing selected examples of (much simpler) artificial systems, it is important for the readers who would not be familiar with molecular machines to first introduce more precisely terms and notions which define their nature and actuation principles.

Achievements in the design and synthesis of artificial molecular machines have been recognized by the 2016 Nobel Prize in Chemistry attributed to Jean-Pierre Sauvage, Sir Fraser Stoddart, and Ben Feringa. ${ }^{[11-13]}$ Following what is now a well-established formalism in the chemical community, ${ }^{[14]}$ molecular machines are (supra)molecular entities in which subcomponents produce controlled and large amplitude movements relative to one another, and which use this mechanical actuation as a functional tool. Molecular machines are divided, following their functioning principles, between switches and motors. Switches are bistable (or multistable) molecules actuated back and forth upon optical, electrical, or chemical stimuli. They influence their surrounding environment as a function of state, and any work produced by the switch at nanoscale in one direction is cancelled when the system is reset to its initial 
state. A particular class of molecular machines has been designed by the implementation of a new type of chemical bond, ${ }^{[15]}$ the so-called mechanical bond, which generates mechanically interlocked molecules such as catenanes and rotaxanes. ${ }^{[16]}$ Here, rings and threads can move between different stations, with a selectively ruled by a set of internal and external thermodynamic parameters, and perform well-defined motions of large amplitude (Figure 1a). ${ }^{[17,18]}$ It should be noted that another class of molecules, namely photochromic switches which make use of local configurational isomerizations - such as azobenzenes - can also be considered, in certain circumstances, as (part of) machines capable of local mechanical actuations, but with more limited amplitudes. Their implementations in responsive materials involving several of their intrinsic switchable properties have been already discussed in the literature. ${ }^{[19]}$

In contrast to mechanical switches, molecular motors move their subcomponents along a nonreversible energy profile, and influence their surrounding environment as a function of their trajectory. ${ }^{[20,21]}$ This very peculiar behavior provides them with the capacity to progressively increase the work they perform by cycling their internal motion when fueled by an external source of energy. The functioning principles of motors at nanoscale are particularly complex because achieving directionality in Brownian motion requires to make use of ratcheting strategies to break spatial and time-reversal symmetries. ${ }^{[22]}$ Light-driven artificial molecular motors have been now designed by synthetic chemists, originally by making use of crowded alkene photoswitches coupled to chiral conformational states and power stroke actuation principles (Figure 1b $(i-i i)) .{ }^{[23]}$ Also, chemically-driven molecular motors capable of biasing Brownian motion have been developed by making use of mechanical bonds and information ratchets mechanisms (Figure 1b (iii)). ${ }^{[24,25]}$ In general, the functional tasks that can be performed using molecular machines have been demonstrated on their own scale (e.g. transport of nanometric cargo, mechanochemical catalysis, molecular pumping). However, the recent literature shows that molecular machines can also be integrated and interfaced with 
other elements to harness their collective motions up to microscopic and even macroscopic scales. This progress report highlights the most remarkable endeavors performed towards the global objective of synchronizing and amplifying individual actuations of molecular machines in larger stimuli-responsive assemblies at surfaces, interfaces, and in 3D materials. Each of the sections will be discussed by the type of molecular machines involved (catenanes, rotaxanes, and light-driven rotary motors).

\section{Molecular machines at surfaces and interfaces}

The extraction of mechanical work from molecules submitted to Brownian motion is one of the important questions related to the functioning principles of nanomachines and to their implementation in materials. 2D platforms are particularly useful to immobilize and align molecular switches and motors in order to avoid complete dissipation of their nano-actuation in the medium, and in order to produce spatially oriented collective motions. In return, molecular machines can provide surfaces and interfaces with mechanically active properties as described hereafter for Langmuir monolayers, self-assembled monolayers, nanoparticles, and mesoporous materials.

\subsection{Langmuir Monolayers}

In 2000, the group of Stoddart successfully achieved the formation of Langmuir films made of dimyristoylphosphatidic acid (DMPA) and tetracationic [2]catenanes which involve $\pi-\pi$ interactions between the cyclophane units. ${ }^{[26]}$ The Langmuir-Blodgett (LB) technique allowed transfer of the films onto quartz surfaces without damage and, as elucidated by UV-Vis and small-angle X-ray scattering experiments, the thickness of these films was in agreement with the one of a single [2]catenane/phospholipid bilayer. Subsequently, the same group demonstrated that Langmuir films build from reversibly redox-switchable cationic 
[2]catenane and DMPA anions could be transferred onto gold or mica surfaces by using the horizontal lifting technique. ${ }^{[27]}$ Current/voltage measurements on the tetra- and hexacationic monolayers using scanning tunneling spectroscopy showed different conduction behaviors between the two states of the [2]catenane which induce different organization of the monolayers as indicated by AFM experiments. These initial results paved the way towards the use of bistable [2]catenanes in molecular electronic devices (Figure 2a). The first electronically reversible switch - known as a molecular switch tunnel junction (MSTJ) - was achieved in 2000 by sandwiching a LB monolayer of bistable [2]catenane $\mathrm{C}^{4+}$ between a polysilicon bottom electrode and a Titanium/Aluminium top electrode (Figure 2a $(i))^{[28]}$ Interestingly, when the device was switched on and off by applying voltages at $+2 \mathrm{~V}$ and $-2 \mathrm{~V}$ respectively (and with a reading at $0.1 \mathrm{~V}$ ), a change in the junction resistance related to the bistability of the molecular machine was observed and could be cycled several times (Figure 2a (ii)). This bistability is related to the presence of two different co-conformations which present different conducting properties: $i$ ) the ground-state one (GSCC), in which the electron-accepting cyclobis(paraquat-p-phenylene) $\left(\mathrm{CBPQT}^{4+}\right)$ ring is located around the electron-donating tetrathiafulvalene (TTF) unit, and ii) the metastable-state one (MSCC), in which the $\mathrm{CBPQT}^{4+}$ macrocycle surrounds the 1,5-dioxynaphtalene (DNP) unit (Figure 2a (iii)). Typically, in MSTJs, the GSCC corresponds to the low conduction state and so-called switch OFF state, while the MSCC related to a high conductance state, assigned as the switch ON state. ${ }^{[29]}$ While being independent on whether charge transport occurs by tunneling or hopping, this $\mathrm{ON} / \mathrm{OFF}$ switching process is related to different charge transfer rates between the two states based on an energy gap hypothesis. ${ }^{[30]}$ This theoretical explanation proved to be valid for MSTJs involving either [2]catenanes and [2]rotaxanes. ${ }^{[31,32]}$ Based on principles similar to the ones used for MSTJs, the groups of Stoddart and Heath reported other kinds of devices with a switching ability related to the bistability of [2] rotaxanes. For instance, a 2D crossbar circuit built from micrometer-scale MSTJs was used to produce a 64-bit random 
access memory with repeatable cycling properties. ${ }^{[32]}$ Few years later, a high-density electronic memory was built from a crossbar circuit containing 160 000 individual MSTJs corresponding each for one bit (Figure 2b). ${ }^{[33]}$ Each MSTJ was made of 200 [2] rotaxane molecules deposited at the cross-section between a bottom silicon and a top titanium nanowire electrodes (Figure 2b $(i)$ ). However, the use of this technology in the next generation of memory devices has been limited due to their limited cycling capacities along with the presence of a large number of defects involving poorly switching bits.

In parallel to these practical applications of [2]rotaxanes, a series of analytical and computational studies have been reported in order to determine which experimental parameters would account for designing the best switchable devices. ${ }^{[34-39]}$ First, it was shown that actuation of [2]rotaxanes can occur using chemical oxidation/reduction even in densely packed LB monolayers, as determined by XPS analysis. ${ }^{[34]}$ Second, the importance of the chemical design was probed, including the presence of the cyclophane ring as well as the length and hydrophilic character of the tails. Third, the Langmuir/LB experimental setups were refined and parameters such as elevated temperature, slow compression rate, and surface pressure below the transition region were shown of importance to reach Langmuir films with high stability and surface viscoelasticity. ${ }^{[35]}$ Molecular dynamic (MD) simulations revealed also that GSCC and MSCC co-conformers lead to similar LB monolayer thicknesses at a same packing area, suggesting that redox switching does not induce a significant reorganization of the monolayer superstructure. ${ }^{[36]}$ However, combining X-ray reflectivity measurements with MD simulations suggested that shuttling of the $\mathrm{CBPQT}^{4+}$ ring from the TTF to the DNP site requires more conformational reorganization within molecular electronic devices than does the reverse process, because of different unit tilt angles. Within MSTJ devices, the presence of bulky hydrophobic stoppers, which can interact with the titanium layer and protect the switching element of the rotaxane, as well as the use of densely packed monolayers are crucial to reach high performance devices. ${ }^{[37]}$ The importance of the bulky 
stoppers (or of covalent bonds) for enhanced device robustness was further confirmed by a DFT study. ${ }^{[39]}$ Interestingly, when a monolayer of these amphiphilic rotaxanes was compressed at $\sim 5 \mathrm{~mm} / \mathrm{min}$, the formation of solitary travelling waves was observed by laser scattering microscopy. This phenomenon was attributed to defects within the monolayer, but its origin remains to be fully elucidated. ${ }^{[40]}$ In parallel to the work developed by Stoddart and Heath, the groups of Zhang and Zhu have shown that the electrical bistability associated with similar [2] rotaxanes can be preserved in multilayers (up to 50) LB films deposited either on HOPG or ITO substrates. ${ }^{[41]}$ Indeed, conducting AFM, STM, and two-terminal junction device studies on several multilayer devices have shown that a threshold voltage (over $1.4 \mathrm{~V}$ ) is necessary to observe a transition from a low-conductivity state to a high-conductivity one, in agreement with the shuttling of the macrocycle from the TTF station to the DNP one. Finally, the group of Credi also reported the formation of devices made by multilayers of nonamphiphilic acid-base switchable [2]rotaxanes transferred onto ITO surfaces. ${ }^{[42]}$ Characterization of the switching properties of the multilayers were performed using cyclic voltammetry in solution. Interestingly, upon exposure to acid and base vapors, electrical switching of the device occurred in a reversible manner over up to two cycles. However, the authors could not fully determine whether the switching process had to be attributed to the shuttling of the macrocycle or to a reorganization of the film at the surface upon $\mathrm{pH}$ stimulation. These seminal works clearly highlight the possibility offered by the bottom-up organization of switchable mechanically interlocked molecules within Langmuir monolayers in order to design synchronous actuation and functional electronic devices therefrom. ${ }^{[43]}$

\subsection{Self-assembled Monolayers}

As an alternative to devices built on LB monolayers, the groups of Stoddart and Zhang studied the electrochemical switching of bistable [2]rotaxane covalently confined as selfassembled monolayers (SAMs) on gold surfaces. ${ }^{[44]}$ Electrochemical measurements involving 
the use of gold nanowires covered with a SAM of [2] rotaxane as working electrode unambiguously showed that the functioning principle of the SAM-based device is almost identical to the one elucidated in LB monolayer, albeit with an increased MSCC $\rightarrow$ GSCC relaxation time accounting for an increased steric confinement within the SAM. Subsequently, the bistability of this [2] rotaxane was used to modulate the localized surface plasmonic resonance (LSPR) properties of nanometer-scale gold nanodisks arrayed on a glass substrate (Figure 2c). ${ }^{[45]}$ The reversible shift of the LSPR peak $(\lambda=690 \mathrm{~nm})$ of the gold nanodisk occurring upon a chemical oxidation/reduction process was found to be directly related to the electrochemical switching between the GSCC and the MSCC co-conformers (Figure 2c (i-ii)). With the aim to build a nanomechanical device which rests on similar actuating principles, a redox-sensitive bistable [3] rotaxane composed of two TTF and two DNP stations, together with two CBPQT rings functionalized with thioctic acid moieties was used to build SAMs on gold-coated microcantilever arrays. ${ }^{[46,47]}$ Upon exposure to a chemical oxidant, a negative deflection up to $35 \mathrm{~nm}$ of the cantilever beam was observed in agreement with a motion of the $\mathrm{CBPQT}^{4+}$ rings from the TTF to the DNP stations leading to a contraction of the mechanically active molecules. Exposure to a reducing agent led to a recovery of the initial position of the [3]rotaxane molecules within the SAMs - and thus of the cantilever - in agreement with an extension process. Importantly, control experiments confirmed that the force measured at the micrometer-scale level of the cantilever results directltly from the concerted actuation of billions of mechanically active molecules switching at the nanometric scale.

In a study combining simulations and DFT calculations, the group of Goddard determined that, at optimal packing conditions, SAMs made of GSCC [2]rotaxanes have a lower surface tension compared to the ones made of MSCC. ${ }^{[48]}$ This theoretical result was in good experimental agreement with water contact angle measurements performed on SAMs modified surfaces, which present a higher hydrophilic character in the oxidized state (MSCC) than in the reduced state (GSCC). Modifications of the wetting properties of surfaces covered 
with mechanically interlocked molecules and motors have attracted the attention of several other research groups. More than 15 years ago, Willner and co-workers reported the modification of a gold surface by SAMs of redox-active monostable rotaxanes consisting of a diiminobenzene $\pi$-donating station, a $\mathrm{CBPQT}^{4+}$ macrocycle, and an adamantane stopper (Figure 2d). ${ }^{[49]}$ Reduction of the CBPQT ring by electrochemical means leads to its shuttling toward the gold surface, resulting in a change of the surface wettability from hydrophilic to hydrophobic as demonstrated by contact angle measurements. Oxidation of the reduced ring restores the initial properties of the surface in a reversible manner over several cycles. Shortly after, the group of Leigh and Zerbetto reported that surface properties can also be modified by the actuation of light-responsive bistable [2]rotaxanes physically adsorbed via hydrogen bonding interactions on a gold surface modified with 11-mercaptoundecanoic acid. ${ }^{[50]}$ Upon exposure to UV irradiation $(253 \mathrm{~nm})$, photoisomerization of the $E$-fumaramide station to the Z-maleamide induces the motion of a tetraamide macrocycle towards the tetrafluorosuccinimide station. Considering the polarophobic character of the fluoroalkane region, UV irradiation leads to the formation of a more polarophilic surface, resulting in a decrease of contact angle measurements performed using diiodomethane or water. Interestingly, a macroscopic motion of a diiodomethane droplet over a flat or $12^{\circ}$-inclined SAM-modified surface could be observed upon irradiation, because of a surface wettability change, which is the consequence of a local change of the rotaxane conformation. More recently, the groups of Unger and Schalley reported surface properties modifications upon chemical stimulation of gold surfaces either covalently decorated by rotaxane SAMs or by supramolecular multilayers of analogous rotaxanes (Figure 2e). ${ }^{[51]}$ Upon addition of chloride anions, the tetraamide macrocycle moves from the diketopiperazine station to the triazole station, and in a reversible manner after precipitation of the chloride anions. This shuttling motion observed in solution by ${ }^{1} \mathrm{H}$ NMR spectroscopy occurs also on rotaxane-modified surfaces, as determined by contact angle measurements. Reversible changes of the surface 
wettability over several cycles can be attributed to the molecular motion of the rotaxanes upon chemical stimulation, as confirmed by angle-resolved NEXAFS experiments.

Based on a very different molecular design, the group of Feringa envisioned the use of lightdriven rotary motors covalently anchored on surfaces in order to modulate their wetting properties. In several fundamental studies, using mostly optical spectroscopies (UV-Vis and circular dichroism), they established that the so-called second-generation of light-driven motors conserve their rotary motion on surfaces such as silicon, mica, quartz, and semitransparent gold film deposited on quartz surfaces. ${ }^{[52-55]}$ Importantly, for motors mounted on gold substrates, the presence of long anchoring linker on the stator part was crucial to achieve the $360^{\circ}$ rotation of the rotor part without quenching the excited state of the motor. ${ }^{[53]}$ Furthermore, several chemical reactions such as amidation, gold-sulfur interactions, silanization or even [3+2] Huisgen copper catalysed azide/alkyne cycloaddition ("click" chemistry) can be used to form these SAMs. Interestingly, for mica surfaces functionalized with motors, molecular "carpets" with a thickness of $\sim 2$ to $2.5 \mathrm{~nm}$ are observed by AFM, which is in agreement with the estimated length of the motor. Upon UV irradiation, morphological changes such as new pinholes or broadening of existing ones are observed, probably arising from a structural reorganization of the motor layer not covalently attached to the mica surface. ${ }^{[55]}$

In order to study possible changes in surface wetting properties, motors functionalized with hydrophilic cyano groups or hydrophobic perfluorobutyl units on their rotor part were grafted by "click" chemistry on quartz surfaces via their stator part. ${ }^{[56]}$ While UV-Vis experiments confirmed the proper functioning of the motors both in solution and on quartz surface, contact angle measurements performed on either the cis or the trans modified surfaces confirmed their different wetting properties depending on whether the substituents are oriented toward or outward the quartz surface. Attempts to modify the surface wettability in situ by UV irradiation did not proceed, probably due to intermolecular interactions between the motors, 
which lower the photoconversion and slow down the thermal isomerization step. To circumvent this problem, a motor decorated with a rigid tripodal stator and a perfluorobutyl side chain on the rotor was covalently self-assembled on a gold surface either as its stable-cis or stable-trans isomer (Figure 2f). ${ }^{[57]}$ Such chemical design prevents any interference between the motors within the SAM, preserves both the photoisomerization and thermal isomerization steps on the surface, and enhance their stability upon prolonged UV irradiation times. Interestingly, upon UV light irradiation, a reversible change by a maximum of $22^{\circ}$ of the contact angle of a water droplet deposited on the motor-modified surface was observed in agreement with a reversible $c i s \leftrightarrow$ trans isomerization of the motor.

Altogether, these examples show that the implementation of molecular machines in SAMs is a particularly rich domain of investigations, and can give access to interesting stimuliresponsive properties. These properties can be either provided by a transduction of the mechanical motion into a modification of a given physical parameter (conductivity, plasmonic resonance frequency, wettability), or they can be provided by a direct mechanical amplification of the nanoactuators when coupled in larger collective motions, and going from the low nanometer range to several dozens of nanometers.

\subsection{Nanoparticles}

As an alternative to n-type poly-silicon bottom electrode, single-walled carbon nanotubes (SWNT) deposited on a silicon wafer have been used to fabricate a molecular switch tunnel junctions (MSTJs) device containing bistable [2] catenanes (Figure 3a). ${ }^{[58]}$ Their structure is similar to the one already used in [2]catenane-based MSTJs ${ }^{[28]}$ excepted that the bipyridinium stations are replaced by diazapyrenium ones, in order to enhance non-covalent interactions between the [2]catenane units and the SWNT layer. Final composition of the device consisted in a layer of DMPA anions followed by a Ti/Al top electrode. This device showed characteristics (hysteretic current-voltage response, device cycling between on and off states) 
similar to the ones recorded for the device based on poly-Si electrode (Figure 3a (ii)). Importantly, control experiments using non redox-active [2]catenane or just the tetracationic diazapyrenium cyclophane confirmed that the bistability of the catenane is responsible for the switching properties of the device. Similar [2]catenanes have also been investigated as redoxactive units on metal nanoparticles (gold, palladium, and platinum) in order to tune their surface and electrochemical properties. ${ }^{[59]}$ The metal nanoparticles were coated with different percentage of [2]catenanes in order to ensure a good solubility in organic solvents. The redoxactive properties of the mechanically interlocked structure were preserved on all metal nanoparticles. Chemical oxidation with iron perchlorate (III) led to an increase of the zeta potential of the nanoparticles, which could be cycled reversibly by chemical reduction with ascorbic acid over several cycles. The use of cyclic voltammetry experiments confirmed that the oxidation and reduction events taking place on the nanoparticles are directly related to the redox-switching properties of the [2]catenane. Similar observations were made using a redoxswitchable [2]rotaxane anchored on gold nanoparticles. ${ }^{[60]}$ These two examples show that [2]catenanes can retain their redox-switching properties on nanoparticles, leading to potentially interesting hybrid actuators.

In 2003, the group of Fitzmaurice reported the functionalization of $\mathrm{TiO}_{2}$ nanoparticles with an electroactive [2]rotaxane containing two different bipyridinium stations separated by a short propyl linker and a crown ether macrocycle as movable unit (Figure 3b). ${ }^{[61]}$ The mechanically interlocked molecule was attached covalently to the nanoparticles thanks to the presence of a tripodal stopper decorated by phosphonic acid groups, thus keeping the orientation of the rotaxane perpendicular to the surface during actuation (Figure $3 b(i)$ ). While ${ }^{1} \mathrm{H}$ NMR experiments could be used to demonstrate the shuttling motion of the ring, cyclic voltammetry was instrumental to demonstrate that one to four electrons can be transferred from the conducting band of the $\mathrm{TiO}_{2}$ to the rotaxane component. Depending on the applied potential, the crown ether macrocycle can move reversibly to the appropriate station. Interestingly, a 
conformational change of the rotaxane occurs upon transfer of four electrons leading to the formation of its fully reduced form, which subsequently requires more positive potentials to reversibly oxidize it (Figure 3b (ii)). Few years later, the same group studied by cyclic voltammetry and spectroelectrochemistry the influence of the flexibility of the linker on the shuttling and electrochemical behavior of the rotaxane. ${ }^{[62]}$ Importantly, in contrast to flexible linkers, rigid ones such as triphenylene units precluded any molecular shuttling as explained by the formation of an electrical double layer at the interface. In 2013, a hybrid device with interesting electronic properties was elaborated by grafting a monolayer of a light-responsive bistable rotaxane non-covalently on a single-layer graphene sheet (Figure 3c). ${ }^{[63]}$ As triethanolamine is required to operate the device, its integration into a microfluidic system allowed its functioning under oxygen-free conditions. When the device was flowed by triethanolamine under light irradiation (i.e. reducing conditions), a large decrease of the drain current of the graphene $(12 \%)$ was observed, resulting from the shuttling of the CBPQT ${ }^{4+}$ ring from the DNP station to the bipyridinium station. Reversible switching of the device occurred by exposing it to oxygen in the dark, and cycling of the device could be repeated several times without apparent loss of its electrical properties (Figure 3c (ii)). The combination of cyclic voltammetry and UV-Vis absorption data allowed determining that the HOMO level of the reduced rotaxane is close to the Fermi level of graphene, facilitating their electronic communication and thus leading to an increase in the resistance of the graphene (Figure 3c (iii)). These data support the fact that, in its reduced state, the device can be stored in a low conductance state providing that it is stored under air-free conditions. More recently, $\mathrm{SiO}_{2}$ nanoparticles decorated with $\mathrm{pH}$-switchable bistable [2] rotaxanes stoppered by a fluorescent 4-morpholinnaphtalimide unit showed interesting optical switching properties both in solution and in the solid state. ${ }^{[64]}$ Upon deprotonation of the ammonium station, the crown ether macrocycle that incorporates ferrocene electron donor units moves to the triazolium station located closer to the fluorescent moiety. This actuation of the mechanical 
bond leads to a quenching of the fluorescence resulting from a photo-induced electron transfer process occurring between the ferrocene and the 4-morpholinnaphtalimide units. Further protonation of the ammonium station causes the ring to shuttle back to its initial position restoring the fluorescence, both in solution and in the solid state.

While [c2] daisy chain rotaxanes have been widely studied as active mechanical components in polymer systems (see section 5), their use in other types of materials remains limited to date. Few years ago, the group of Tian reported the reversible actuation of gold nanoparticle dimers covalently connected by a [c2]daisy chain rotaxane. ${ }^{[65]}$ Motion was observed by plasmonic resonance scattering spectroscopy when one nanoparticle physically adsorbed on an ITO substrate was connected via the mechanically interlocked molecule to a second nanoparticle. Combining simulation with dark-field microscopy experiments allowed determining that a reversible motion of $1.17 \mathrm{~nm}$ occurs over several cycles on surface between the nanoparticles.

Examples of a successful immobilization of a light-driven unidirectional rotary motor on gold nanoparticles dates back to 2005 but are very few and limited to molecular scale motions. ${ }^{\text {[66] }}$ Upon photoisomerization around the double bond, a repetitive and unidirectional $360^{\circ}$ rotary motion occurs on the gold nanoparticles as determined by extensive circular dichroism and ${ }^{1} \mathrm{H}$ and ${ }^{13} \mathrm{C}$ NMR spectroscopies. ${ }^{[67]}$ These experiments confirmed that both photochemical and thermal processes are preserved on surfaces, albeit with a two-fold increase of the half-life time for the thermal helix inversion at room temperature.

These examples show the first implementations of molecular machines to modify the surfaces of various types of nanoparticles in order to access electroactive or plasmonic hybrid materials. They also suggest that many other possibilities and interesting works will be done in a soon future which will exploit their full potential in such configurations, for instance by taking the opportunity offered by molecular motors to mechanically propel nanoparticles. 


\subsection{Mesoporous Materials}

Mesoporous nanoparticles (MSNPs) of large sizes (100-800 nm), capable of storing guest molecules, and functionalized with switchable molecular machines, have emerged as potential drug delivery systems named as nanovalves. ${ }^{[68,69]}$ In particular, when functionalized with bistable rotaxanes, the movement of the macrocycles from one station to another helps to open and close the nanopores resulting in a controlled loading and release of guest molecules (Figure 3d (iv)). Over the years, several groups have examined the possibility to use different stimuli (such as redox, light, or $\mathrm{pH}$ ) to produce responsive nanovalves. In 2005, the groups of Stoddart and Zink reported the first active MSPNs involving redox-sensitive [2] rotaxanes with a $\mathrm{CBPQT}^{4+}$ ring shuttling between a tetrathiafulvalene station and a dioxynaphtalene station (Figure 3d $(i)) .{ }^{[70,71]}$ Within a four-step sequence relying on the chemical oxidation/reduction of the TTF station, the nanoparticles could be sequentially loaded and unloaded with luminescent guests in a controlled manner, as determined by fluorescence microscopy. Further studies demonstrated the importance of the length of the anchoring linker (the shorter the better) to limit the leakage of the nanovalves while the distance between the stations had almost no influence. ${ }^{[71]}$ Alternatively, $\mathrm{pH}$-sensitive bistable [2]pseudorotaxane consisting of an axle containing 1,6-hexanediammonium and 1,6-bis(pyridinium)hexane recognition sites, and a cucurbit[6]uril as macrocycle were successfully anchored on MSNPs (Figure 3d (ii)). ${ }^{[72]}$ Under neutral or acidic conditions, the macrocycle surrounds the bisammonium station located close to the NP, thus blocking the pores. However, upon raising the $\mathrm{pH}$ in a range from 8 to 10 , deprotonation occurs inducing the motion of the macrocycle to the bis-pyridinium station and subsequently leading to the release of the loaded guest molecule. In solution, controlled release of guest molecules was achieved by adjusting the $\mathrm{pH}$ and reloading of the MSNPs was possible albeit with reduced loading capacity.

Alternatively, in order to consider MSNPs for in vivo applications, light-responsive systems, initially resting on the use of non-bistable pseudo-rotaxanes, ${ }^{[69]}$ have been developed. More 
recently, the groups of Stoddart and Zink reported the successful light-activation of such MSNPs with azobenzene and adamantane as stations and $\alpha$-cyclodextrin as a shuttling ring (Figure 3d (iii)). ${ }^{[73]}$ In 2012, the group of Zhao described the first in vivo application of lightsensitive MSNPs functionalized with [2]rotaxanes consists of an $\alpha$-cyclodextrin ring shuttling between a triazole in its closed form and an azobenzene unit in its opened form. ${ }^{[74]}$ Upon irradiation with visible light or heating at $65{ }^{\circ} \mathrm{C}$, cis $\rightarrow$ trans photoisomerization of the azobenzene causes an opening of the pores in order to load the MSNPs with a fluorescent dye and a therapeutic agent, which were efficiently trapped without apparent leakage after UV irradiation. Fluorescent measurements performed either in solution or in vivo with optically transparent zebrafish larvae confirmed the efficient release of the guest molecules upon concomitant visible light and thermal activation of the MSNPs. Interestingly, when zebrafish larvae presenting heart failure were treated with the curcumin-loaded MSNPs, an improvement of their heartbeats and ventricle contractility was observed, in agreement with the efficient release of the drug after incubation for 1 hour at $37{ }^{\circ} \mathrm{C}$. This example demonstrates the enormous potential offered by mesoporous nanoparticles decorated with rotaxane units for efficient drug delivery systems. In a subsequent work, NIR irradiation at $808 \mathrm{~nm}$ of the same rotaxane immobilized on gold nanorod-mesoporous silica core-shell hybrids causes the $c i s \rightarrow$ trans photoisomerization of the azobenzene, which was reversibly switched over several cycles with UV light (Figure 3e). ${ }^{[75]}$ As evidenced by confocal imaging, efficient release of doxorubicin, an anticancer drug, within the heads of zebrafish embryo occurred after illumination in the NIR region (Figure $3 \mathrm{e}(i i-i v)$ ), which allows efficient light penetration and low cytotoxicity in living tissues.

To the best of our knowledge, only one example by Feringa and Michl reports the inclusion of unidirectional light-driven molecular motors in mesoporous materials made of a hexagonal tris $\left(o\right.$-phenylene)cyclotriphosphazene (TPP) crystal host. ${ }^{[76]} \mathrm{X}$-ray diffraction along with ${ }^{31} \mathrm{P}$ and ${ }^{13} \mathrm{C}$ solid-state NMR experiments confirmed the formation of inclusion compounds, with 
motors standing on the facets of the disc-shaped nanocrystals. ${ }^{1} \mathrm{H}$ NMR and UV-Vis absorption studies further established that neither surface confinement nor density of surface coverage are affecting the rotary motion of the molecular motors within the crystal and over more than 20 unidirectional rotary cycles.

These few seminal works foreshadow the opportunities of using molecular machines in stimuli-responsive mesoporous materials. Nanomachines have already proved to function in such crowded 2D environments, and they have led to original results for (in vivo) drug delivery applications. But many other implementations could be envisioned based on the mechanically gated access to such high surface area materials, for instance in catalysis and sensing.

\section{Molecular machines in self-assembled 3D materials}

The spontaneous bottom-up fabrication of 3D functional materials by programmed supramolecular self-assembly techniques combines molecular scale precision and easy implementation. Because collective motions of molecular machines can benefit from such an ordering, new ideas recently emerged in the literature to confer mechanically active building blocks with self-assembly properties. As illustrated below, this approach has been successful to amplify the movements of molecular machines up to the actuation of macroscopic objects.

Responsive 3D self-assembled materials built on switchable rotaxanes present the interesting potential to modify their supramolecular structure - in shape or volume - by synchronized mechanical actuations, but they remain limited to only few examples in the literature to date. In 2010, the groups of Chen and Chiu reported the discovery of the first organogelator based on a [2]rotaxane. ${ }^{[77]}$ The gelation properties of this molecular machine originate from the presence of a free urea station involved in intermolecular hydrogen bonding interactions leading to the formation of networks of bundled fibres, as determined by TEM and AFM imaging. Upon addition of a base or a strongly-binding anion, disruption of the gel phase 
occurs as a result of the shuttling of the macrocyclic unit over the urea moiety, which thus alters their hydrogen-bonds networks. More recently, the group of Chung reported a similar strategy to achieve a sol-gel transition in organic solvents using an amphiphilic calix[4]arenebased [2]rotaxanes. ${ }^{[78]}$ However, in this case, SEM imaging suggests that a 3D arrangement of nanosphere aggregates obtained by a combination of various supramolecular interactions $(\pi-\pi$, H-bonds, hydrophobic) leads to the formation of gelified structures, which can be disrupted in a reversible manner upon addition of base. In a more fundamental study, the group of $\mathrm{Qu}$ reported that acid-base actuation of an amphiphilic [2] rotaxane can lead to changes in morphologies from spherical vesicles to worm-like micelles. ${ }^{[79]}$ Shuttling of the macrocyclic unit from the secondary ammonium station to the triazolium one induces a change in packing parameters between the hydrophobic and hydrophilic blocks, which leads to the mesoscopic differences observed by TEM. However, to the best of our knowledge, this promising approach has not yet been implemented to build responsive materials.

Self-assembled 3D materials built from unidirectional molecular motors have emerged only very recently. ${ }^{[80-85]}$ In 2016, the group of Feringa reported the synthesis of two asymmetric molecular motors having different rotation frequencies and bearing one hydrophilic ammonium side chain on the upper part and two hydrophobic alkyl chains on the lower part (Figure 4a) ${ }^{[80]}$ Both motors in a 1/1 mixture with DOPC were found to produce well-defined nanotubes in water. Interestingly, while the fast motor (half-life time of 40 ns at $20^{\circ} \mathrm{C}$ ) did not produce any morphological change upon light irradiation, the slower one $\left(270 \mathrm{~h}\right.$ at $\left.20{ }^{\circ} \mathrm{C}\right)$ gave rise to vesicles which could be reversibly transform into nanotubes upon heating (Figure 4a (iii-viii)). More recently, a hydrophobic second-generation motor, which does not show any rotation in water, could be dissolved in SDS micelles. ${ }^{[84]}$ The motor remained stable for several days, and functions over a large $\mathrm{pH}$ range (2 to 10) to fully rotate up to 13 cycles within the self-assembled nanostructures and showing a photo- and thermochemical behaviour similar to the ones recorded in hexane (half-life of 4.93 min within micelles vs. 
3.17 min in hexane). In 2018, the same group reported the hierarchical self-assembly in aqueous media of a different amphiphilic molecular motor that features a hydrophobic chain on the upper part and carboxylic acid end groups on two side chains of the lower part (Figure 4b). ${ }^{[81]}$ While this motor gives rise to individual nanofibers with a high aspect ratio in water, it can further assemble into a macroscopic string consisting of aligned bundles when it is drawn into a $\mathrm{CaCl}_{2}$ aqueous solution, as a result of electrostatic interactions between $\mathrm{Ca}^{2+}$ ions and carboxylate groups. Photo-irradiation with UV light of these strings either in water (Figure 4b (ii)) or in air (Figure $4 \mathrm{~b}(\mathrm{iii}))$ leads to their bending at a saturated flexion angle of $90^{\circ} \mathrm{C}$ in the direction of the light source within one minute. A series of in situ SAXS measurements revealed that photo-isomerization of the motors induces structural disordering of the local packing arrangement of the nanofibers and increasing of their diameter as they are isomerized from a stable state to an unstable one (Figure $4 \mathrm{~b}(i \mathrm{v})$ ). This change at the nanometric scale leads to a contraction of the nanofibers over their long axis as the total volume of the selfassembled structure remains unchanged during the light irradiation process. Importantly, for all the aforementioned examples, reversibility of the systems, and in particular of the bending process, can only occur thermally because of the high energy barrier necessary for the thermal helix inversion process.

The same group subsequently studied the influence of metal ions and side chain lengths on the hierarchical self-assembled structures and their functioning. ${ }^{[82]}$ Interestingly, while all metal chlorides led to nanofiber formation, only magnesium ions produced bundles of aligned nanofibers with a similar but slightly lower degree of alignment and a closer packing compared to the strings obtained with calcium ions as determined by complementary spectroscopic, scattering, and microscopy experiments. The absence of alignment for barium and mono-charged ions and the lower degree of alignment recorded for scandium, strontium, and beryllium ions suggested the importance of the carboxylate/metal ions binding constants in the hierarchical ordering of the self-assembled structures. Importantly, the actuation speed 
of the strings was found to be directly related to the alignment of the nanofibers and thus to the strength of the electrostatic interactions involved in the self-assembly process. The length of the side chains also influenced the macroscopic response of self-assembled strings as they affect the degree of alignment and actuation speed of the individual nanofibers. Very recently, the same group showed that $\mathrm{Fe}_{2} \mathrm{O}_{3}$ nanoparticles can be grown at the surface of individual motor-containing nanofibers when the carboxylic acid end groups are replaced by histidine residues (Figure 4c). ${ }^{[83]}$ However, the shear flow strategy involving a $\mathrm{CaCl}_{2}$ solution proved ineffective to induce alignment of the individual nanostructures, mainly because of the involvement of the histidine carboxylic unit in the mineralization with ferric ions. Nevertheless, unidirectionally aligned strings were obtained by this strategy using a 1:2 ratio of hybrid nanofibers containing iron nanoparticles and classical motor-amphiphile nanofibers. Upon photoirradiation, the hybrid material showed actuation at a saturation flexion angle of $90^{\circ}$ towards the light source within 25 seconds. SAXS experiments confirmed that bending of the hybrid structures occurs though a mechanism similar to the one described previously. Interestingly, the presence of $\mathrm{Fe}_{2} \mathrm{O}_{3}$ nanoparticles allowed for a magnetic control of the bending motion at the macroscopic scale. This property was then explored to carry out, at the macroscopic scale, a cargo transport experiment, which consists in a series of translocation and load/unload events, by taking advantage of orthogonal magnet and light stimuli (Figure $4 \mathrm{c}(i i))^{[83]}$

A recent study by the groups of Feringa, Wezenberg and Browne has also shed light on the potential of using molecular motors in the solid state. ${ }^{[85]}$ They designed and synthesized a metal-organic framework (MOF), using a solvent-assisted linker exchange (SALE) strategy, ${ }^{[86]}$ in which pyridine-functionalized molecular motors act as organic struts that connect layers made of zinc nodes bridged by tetracarboxylic acid linkers (Figure $4 \mathrm{~d}(i)$ ). Xray powder diffraction, Raman and NMR spectroscopies confirmed the integrity of the MOF upon light irradiation in DMF. Rotation of the motors within the MOF upon photoirradiation 
and during thermal relaxation was characterized by changes in the Raman spectrum similar to the ones recorded for the molecular motor alone in solution (Figure 4d (ii)). Interestingly, the motors showed half-life time in the solid state comparable to the ones monitored in solution and without affecting the stability of the framework, as demonstrated by cycling experiments. Such dynamic crystalline frameworks would find applications as light-responsive materials for environmental science (storage and diffusion of gases for instance) or even as miniaturized pumping modules in microfluidic devices.

Overall, these studies clearly demonstrate that molecular machines can be associated in various self-assembled materials going from soft-matter systems to more crystalline objects. They also show that their actuation properties can be preserved in these configurations, and that in turn their motions can influence the stability and the morphology of the self-assembled structures in which they operate. This can lead to structurally dynamic object which can change their association state or their shape (such as in diluted mesophases), but can also actuate objects up to the macroscopic scale. As such, they would be of great interest in the future design of artificial muscles.

\section{Molecular machines in liquid crystal materials}

Order parameters in liquid crystals can in principle support and/or translate the directed motions of molecular machines towards higher length scales in collective systems. It is indeed well-known that, because of their fluid character and of their capacity to generate phase transitions at low activation energies, they can produce fast and long range spatial rearrangements of their mesophases by minimal mechanical stimulations. Therefore, this situation appears ideal to translate the nanoscale actuation of molecular machines, and this was confirmed by a string of successful studies which illustrate the potential of this approach. To the best of our knowledge, the formation of liquid crystalline materials based on catenane is limited to the collaborative work reported by Sauvage and Kato more than ten years 
ago. ${ }^{[87,88]}$ They synthesized a [2]catenane with a mesogenic side chain based on the wellknown fluorosubstituted cyclohexylbiphenyl moiety, ${ }^{[89]}$ and bearing one phenanthroline station within each macrocycle, which can thus be locked by adding copper (I) (Figure 5a (i)). Above a similar glass transition temperature $\left(\sim 68^{\circ} \mathrm{C}\right)$, both the free catenane and its copper complex exhibited a smectic liquid crystalline phase which results from a layered parallel arrangement of the mesogenic cores sandwiching a more flexible layer of the [2]catenane linkages (Figure 5a (ii)). ${ }^{[87]}$ Although the presence of copper did not influence the kind of LC phase observed, it drastically increased its stability as the isotropization temperature increased by $30^{\circ} \mathrm{C}$ (from 117 to $147^{\circ} \mathrm{C}$ ). This enhanced stability was attributed to an increased rigidity of the catenane layer because of electrostatic interactions. The interlocked topology of the molecular switch was found to increase the thermal stability of the LC phase compared to the free macrocycle, but it had no influence on the molecular organization in the LC phase, as determined by SAXS experiments. ${ }^{[88]}$ Obviously, a straightforward extension of this work would consist in developing a version involving bistable catenanes in order to confer a related stimuli-responsive character to the liquid-crystal mesophase.

In parallel to their work with the group of Sauvage, Kato and co-workers, in collaboration with the group of Stoddart, reported the first example of a liquid crystalline bistable [2]rotaxane (Figure $5 b(i)$ ). ${ }^{[90]}$ This molecule consists in an axle bearing one tetrathiafulvalene (TTF) and one 1,5-dioxynaphtalene (DNP) unit as stations surrounded by a cyclobis(paraquatp-phenylene) $\left(\mathrm{CBPQT}^{4+}\right)$ ring and two mesogenic stoppers identical to the ones used in the LC catenanes ${ }^{[87,88]}$ and which are known to be electrochemically inactive. This compound displayed a smectic LC phase between 10 and $150{ }^{\circ} \mathrm{C}$ as determined by using a combination of differential scanning calorimetry (DSC), microscopy, and scattering experiments. Importantly, the authors demonstrated that shuttling of the macrocycle from one station to another, i.e. over an estimated distance of $1.4 \mathrm{~nm}$, could be achieved in a reversible manner in the LC state by using an electrochemical stimulus, as determined by cyclic voltammetry 
experiments. ${ }^{[91]}$ In an electrochromic cell, when applying positive potentials up to $+1.6 \mathrm{~V}$, changes in colour from brown to pink were observed and correlated with the oxidation of the TTF unit, shuttling of the CBPQT macrocycle and the formation of a new charge transfer complex with the DNP station (Figure 5b (ii)). This colour change occurs within $10 \mathrm{~s}$ upon oxidation while requiring slightly longer time (40-50 s) for the reduction, probably because of attractive interactions between the DNP station and the CBPQT ring. This example represents one of the first examples perceptible by the naked eye, which demonstrated how the redoxdriven shuttling at the molecular scale can lead to modifications of a physical property at the macroscopic scale of the material. Recently, the groups of Sevick and Williams reported a theoretical study, which proposes that an inefficient system, i.e. with no quantitative switching of the mechanical bond, could lead to a macroscopically observable phase transition from nematic to isotropic. ${ }^{[92]}$ Although this study has not yet been experimentally demonstrated, it suggests that a blend of LC molecules with and without a mechanical bond could be sufficient to observe such phase transition. However, so far, all examples of mechanically interlocked molecules with liquid crystalline properties have failed to induce crystalline phase change (for instance, from columnar to lamellar) upon actuation of the mechanical bond.

Besides, two approaches have been envisioned to produce liquid crystalline materials based on molecular motors. The most studied one consisted in using the molecular motor as chiral doping agent in a LC solvent. For instance, in the early 2000s, a commercially available LC phase doped with a low weight percent of first-generation rotary motor was used to demonstrate that the unidirectional rotary process of overcrowded-alkene motors is retained in this particular environment. ${ }^{[93]}$ The high helical twisting power (HTP, $\beta$-value) of the stable $(P, P)$-trans isomer suggests that this molecule is efficient to induce a chiral orientation in the LC phase. Furthermore, the low $\beta$-values of the unstable $(P, P)$-cis and $(M, M)$-trans isomers encouraged the use of this light-responsive unidirectional motor to achieve colour tuning of a 
liquid crystalline film with light irradiation. When a spin-coated LC film of E7 doped with 6.16 weight $\%$ of the motor in the $(P, P)$-trans configuration is irradiated at a wavelength above $280 \mathrm{~nm}$, an elongation of the pitch of the LC phase and a bathochromic shift of the reflection wavelength are induced, leading to a gradual change in colour of the film from violet to red for increasing irradiation times, and as observed by the naked-eye. These observations account for the presence, upon light irradiation, of $(P, P)$-cis and $(M, M)$-trans isomers with lower $\beta$-values than the $(P, P)$-trans. Upon heating at $60{ }^{\circ} \mathrm{C}$, thermal helix inversion of the $(M, M)$-trans isomer into the $(P, P)$-trans configuration occurs, leading to a hypsochromic shift of the reflection wavelength and recovery of the initial colour. A similar behaviour was observed for a LC film doped with a molecular motor of the second generation (Figure 6a). ${ }^{[94]}$ These examples demonstrate the possibility to change the optical properties of a material by transducing at macroscopic scale the molecular motion of motors through molecular reorganization of the mesogens in the LC matrix.

In 2006, the group of Feringa reported the possibility to rotate microscale objects such as micrometric glass rods on a LC film doped with 1 weight $\%$ of a second-generation lightdriven molecular motor. ${ }^{[95,96]}$ This film displays a polygonal fingerprint texture typical of cholesteric phases, which can reorganize upon light irradiation at $365 \mathrm{~nm}$ in a unidirectional rotational manner dictated by the helicity of the motor and can resume when the light is off, with a rotation taking place in the opposite direction. In-depth analysis of the system confirmed that the direction of rotation of the LC film is directly related to the change of the HTP of the motor $(\Delta \beta)$ occurring during its molecular state, with negative and positive changes of $\beta$-values during photochemical isomerization or thermal helix inversion, and leading to clockwise and anticlockwise rotation respectively. Importantly, in response to chirality change of the motor, rotation of the LC phase is accompanied by changes of its cholesteric pitch, which requires a rotational reorganization of the mesogens perpendicular to the cholesteric helix axis. A plausible mechanism to explain this pitch change was proposed, 
based on changes in helix axis direction, which would lead to changes of local directors angle and thus induce local reorganization to align regions with similar directors (Figure 6b (ii)). ${ }^{[96]}$ In addition, the LC film presents regular reliefs of $\sim 20 \mathrm{~nm}$ height and $5.5 \mu \mathrm{m}$ periodicity at its surface which orientation is modified by the topological changes of the motor induced during either the photochemical isomerization or the thermal helix inversion (Figure $6 \mathrm{~b}(i)$ and $(i i i)$ ). The reorganization of these reliefs during photochemical and thermal steps was proposed to induce a torque on the glass rod placed over the film leading to its microscopic motion. It is important to clarify that here the dynamic rotations obtained in the microphases are not a direct consequence of the rotation of the motor - that would be related to its trajectory -, but rather a consequence of its change of state, as it could be generated by simple switches. ${ }^{[97]}$ In a study combining both theoretical and experimental investigations, a more detailed mechanism describing the role of each contributor has been proposed to explain how the molecular rotation of the motors is at the origin of the rotational reorganization observed at the mesoscopic scale. ${ }^{[98]}$ To sum up, 1) the large and quite fast rotational reorganization of the LC phase is directly related to the preferred orientational behaviour of the motor isomers within the LC matrix and to their efficient twisting capability; 2) the interplay between the chirality of the motor and the elastic properties of the LC matrix strongly influences the photochemical process which control the rotational reorganization, and 3) photoisomerization of the motors induces unwinding of the cholesteric helix, thus leading to texture modulation rotation under light irradiation. In 2013, the group of Katsonis demonstrated experimentally that the kinetics of the motors, which is directly related to its structure, is not affected by the LC matrix but it instantaneously determines the relaxation rate of the cholesteric helix. ${ }^{[99]}$ Thus, by fine-tuning the molecular structure of the motor, it becomes possible to accelerate the helix inversion of light-responsive liquid crystals. Overall, these works highlight the importance of chemical design for both the LC matrix and the switchable motor to enable the production of smart light-responsive materials. 
Recently, the group of Yang took advantage of light-responsiveness of a second-generation rotary motor embedded within a rod-like LC matrix to produce reversible dynamic LC diffraction grating on surfaces (Figure 6c). ${ }^{[100]}$ The system was designed so that the helical axis of the LC phase is perpendicular to the substrate resulting in a parallel alignment of the molecules to the substrate. Upon irradiation at $365 \mathrm{~nm}$, which induces photoisomerization of the motors, a decrease of the pitch of the LC from 2.0 to $0.5 \mu \mathrm{m}$ was observed, resulting in the formation of opaque regions arising from the focal conic texture of the random helical domains. Interestingly, the authors subsequently designed surfaces consisting of alternating stripes of covalently polymerized LC networks to stabilize the helices perpendicular to the surface and free helical structures made of motors and LC host (Figure 6c (ii)). While this surface remained transparent at the thermostationary state, exposure to UV induced the formation of a binary pattern made of alternating transparent and opaque regions at the photostationary state, because of alternating planar and focal conic configuration of the LC matrix (Figure 6c (iii-iv)).

Compared to the previous examples, an alternative approach to produce light-responsive LC materials based on molecular motors consisted in covalently grafting a unidirectional rotary motor at the chain end of a LC polymer and studying a possible transmission of chirality from the macromolecular level of the polymer to the supramolecular one. In a fundamental study, Pijper and Feringa demonstrated that the chiral information present in a molecular motor can be used to induce chirality within a covalently attached racemic polymer strand. ${ }^{[101]}$ Based on this work, a modified motor, which behaves as a photoresponsive chiroptical switch between two thermally stable states, was attached by its upper part to the terminus of a LC polymer, namely a $\operatorname{poly}(n$-hexyl isocyanate) (PHIC) (Figure 6d $(i)) .{ }^{[102]}$ The $\left(2^{\prime} S\right)-(P)$ state of the switch favors the $M$ helical conformation of PHIC, which can be fully switched to the $P$ helical conformation upon irradiation at $365 \mathrm{~nm}$ leading to the $\left(2^{\prime} S\right)-(M)$ state of the switch. Complete reversibility of the helical sense of the polymer is then achieved by irradiation over 
$480 \mathrm{~nm}$. Starting from a LC phase containing $30 \%$ weight of $\left(2^{\prime} S\right)-(P)-\mathrm{PHIC}$ in toluene, UV light irradiation induced an elongation of the pitch of the cholesteric liquid crystalline phase until the cholesteric lines vanished ( $45 \mathrm{~min}$ ), due to the presence of equal amounts of $M$ and $P$ helical polymers in the LC film (Figure 6d (ii)). However, prolonged irradiation times (up to $150 \mathrm{~min}$ ) led to a recovery of the cholesteric texture with a shortening of the pitch length, indicating that $M$ helical polymers become predominant over $P$ ones. Interestingly, a similar behaviour was observed starting from a LC film made of (2'S)-(M)-PHIC but longer irradiation times (up to $24 \mathrm{~h}$ ) were required to reach the photostationary state mixture consisting mainly of $P$ helical polymers. In addition, both systems proved to be fully reversible by thermal reorganization of the system in the dark (Figure $6 \mathrm{~d}$ (iii)) and could be cycled several times.

Overall, it has been demonstrated in many examples that molecular machines including catenanes, rotaxanes, and light-driven rotary motors can be associated with liquid crystal materials either by direct chemical coupling to the mesogen, or by using the machine as a chiral dopant. Interestingly, the transfer of chirality using molecular motors allows reaching light-responsive LC materials based on the amplification of a chiral event from the molecular (to the macromolecular) and up to the supramolecular scale. As many technological applications rely on LC materials, there is no doubt that new interesting outcomes based on these approaches will emerge in the future. In addition, an important complementary direction that could be envisioned would consist in coupling molecular machines with oriented selfstanding materials such as liquid crystal elastomers.

\section{Molecular machines in polymers}

By coupling molecular machines to polymers, one can dream of approaching the iconic dynamic structure of muscular fibers, which are made - in a simplistic view - of a combination of motors in an oriented polymer network. In this section, we will discuss more 
generally the various approaches, which have been pursued recently in coupling artificial machines with macromolecules and supramolecular polymers in order to confer them with stimuli-responsive mechanical properties.

The amplification of molecular motion using catenanes was achieved only recently. In 2017, the group of Rowan reported a new synthetic approach involving formation of a supramolecular polymer, ring-closing metathesis, and demetallation as key steps in the preparation of poly[n]catenanes that were obtained in reasonable yields after purification by preparative gel permeation chromatography (Figure 7a). ${ }^{[103]}$ The metalloresponsive properties of the poly[n]catenanes were first investigated in solution showing a higher hydrodynamic radius for the metallated polycatenane compared to the metal-free polycatenane (Figure 7a (ii)). In the bulk, DSC analysis of the glass transition temperature of demetallated poly[n]catenane was found to be $97{ }^{\circ} \mathrm{C}$ while it increased over $160{ }^{\circ} \mathrm{C}$ for the metallated poly[n]catenane (Figure 7a (iii)). These observations are in good agreement with an increased rigidity of the metallated polymer due to the BIP ligands/Zn ions interactions. This work, which represents the first example of amplification of motion with catenane in a polymer, could be of potential interest to create elastomers with flexibility but toughness.

An adaptive gel was also prepared using thiol-ene click reaction between bisvinyl[2]catenane and thiol-modified polyethylene glycol (Figure $7 \mathrm{~b}(i)) .{ }^{[104]}$ In this work, the catenane moiety acts as a crosslinker, which provides rigidity to the material due to the presence of intercomponent hydrogen bondings that restrict the motion of mechanically interlocked moieties. At low $\mathrm{pH}(<7)$ or for high temperatures, i.e. when hydrogen bonding interactions are broken, the gel becomes less rigid as determined by storage and loss moduli measurements (Figure $7 \mathrm{~b}(\mathrm{ii})$ ). Tensile stress-strain measurements corroborated the adaptive character of the gel with a higher tensile modulus and breaking stress recorded for the more rigid Gel 1, in agreement with a more constrained material (Figure $7 \mathrm{~b}(\mathrm{iii})$ ). 
The development of polymers incorporating (pseudo-)rotaxanes units within their backbone has been studied for more than 30 years. ${ }^{[105]}$ Such polymers consists mainly of two different families including main-chain poly(pseudo-)rotaxanes and side-chain poly(pseudo-)rotaxanes. Over the years, they have been designed to produce materials with particular gliding topologies and/or physical properties. ${ }^{[106,107]}$ For instance, the groups of Stoddart and Grzybowski reported the use of a polymethacrylate functionalized with bis-pyridinium macrocycles on the ester side chains to selectively capture and release nanoparticles. ${ }^{[108]}$ When the polymer was immersed in a mixture of silver and gold nanoparticles decorated with TTF and naphthalene ligands respectively, chemical oxidation of the TTF units led to a particular optical signature consistent with the presence of silver nanoparticles in solution and with the precipitation of gold nanoparticles with the polymer. Reduction with ascorbic acid allowed the reversibility of the process, leading to an optical signature characteristic of the presence of the gold nanoparticles in solution and precipitation of the silver ones. In this work, the motion related to the threading/dethreading event at the molecular scale is read out at the macroscopic scale by a typical optical change, but breakage of the mechanical bond is mandatory to achieve the desired physical property. Following a similar approach, the group of Huang reported the synthesis of a main-chain polypseudorotaxane based on host-guest recognition units (crown ether/ammonium) to produce a gel-like material, which presents stimuli-responsive (temperature or $\mathrm{pH}$ ) gel-sol transition properties. ${ }^{[109]}$ This work has certainly participated in paving the way to the development of sol-gel materials based on (pseudo-)rotaxane structures, but the observed macroscopic physical property here again arises mainly from a depolymerisation event rather than a real actuation of the mechanical bond at the molecular level. More recently, main-chain polyrotaxanes such as cyclodextrins (CDs) threaded around a polyethylene glycol chain have been designed as a new type of selfhealing materials. ${ }^{[110]}$ In this work, the presence of hydroxypropylated side chains on the CDs, which could covalently bind polyacrylamide chains incorporating side-chain boronate 
linkages, allowed the synthesis of cross-linked materials with interesting self-healing properties. However, the main contributor to this particular property is the reversible dynamics of the boronic acid bond with the mechanical bond having only a slight influence on the physical self-healing ability of the material. $\mathrm{Xu}$ and co-workers also reported recently how the mobility associated with a mechanical bond can provide a soft membrane with high proton conductivity. ${ }^{[111]}$ In this case, a main-chain poly(crown ether) was decorated with ammonium axles incorporating 1-naphtol-3-sulfonic acid stoppers. Interestingly, when the ammonium units were deprotonated and acylated, the absence of interactions with the crown ether at temperature above $60{ }^{\circ} \mathrm{C}$ mainly favoured the mobility of this axle and thus enhanced proton transfer conductivity across the polymeric membrane. In this case, while the mobility of the mechanical bond is important to reach the desired physical property, it cannot be controlled by its precise actuation. Reaching materials with switching capabilities associated to a controlled actuation of the mechanical bond has been particularly challenging with [ $n]$ rotaxanes and, to the best of our knowledge, examples remained very limited. ${ }^{[112-114]}$ The first one was reported in 2011 by the groups of Stoddart and Heath, who synthesized bistable redox-sensitive poly[n]rotaxanes with 1,5-dioxynaphtalene and TTF stations for bispyridinium macrocycles, in order to construct a solid-state switch. ${ }^{[112]}$ The polymer film was sandwiched between a gold (or a doped poly-Si) bottom electrode and a metallic gold/titanium top electrode. Interestingly, only the device containing one silicon electrode showed switching properties and could be cycled up to 20 times between the on and off conductance states, however with a decreased current level probably due to a degradation of the polymer with voltage. The electrical properties observed for this polymer are very similar to the ones recorded for devices built by integrating the individual molecular machines at interfaces (see section 2), thus suggesting that the collective motion of the rotaxane units at the polymer scale are responsible for the electronic properties of the devices. 
More recently, two groups reported independently the formation of soft materials based on cyclodextrin-based polyrotaxanes with shape recovery and actuating properties, respectively. ${ }^{[113,114]}$ On one hand, the group of Ke synthesized millimetre-sized polyrotaxane monoliths by successive 3D printing and photo-polymerization of triblock copolymer micelles which can thread $\alpha$-CD guests (Figure $7 c(i)) .{ }^{[13]}$ In water, the presence of rigid crystalline domains made of several hydrogen-bonded CD rings leads to mechanically robust materials, while, in DMSO, the absence of hydrogen bonds give rise to smeared hydrogels with low mechanical robustness. Importantly, soaking the monolith back in water induces shape recovery due to reorganization of the $\mathrm{CD}$ at the molecular level. Interestingly, the chemical energy (solvent exchange) necessary to induce macroscopic shape memory properties can be harnessed to produce mechanical work, as demonstrated by lifting a US dime or an aluminium plate (Figure 7c (ii)). In this work, control over the cyclodextrin arrangement within the polyrotaxane chain dictates the shape recovery properties observed at the macroscopic level. On the other hand, Harada and co-workers reported the actuating properties of a polymer network in which cyclodextrin/azobenzene interactions act as reticulating points (Figure $7 \mathrm{~d}$ $(i)) .{ }^{[114]}$ Upon irradiation with UV light, an azobenzene unit is switched from the trans isomer to the cis, which dethread from the $\alpha$-CD cavity, whereas irradiation with visible light leads to the reversible process. Interestingly, such behaviour is also observed within the hydrogel and xerogel materials, resulting in a decrease of the Young modulus after UV irradiation (Figure $7 \mathrm{~d}(i i))$. This mechanical response is directly related to the actuation of the mechanical bond, as the $\alpha-C D$ can freely move along the polymer chains when the azobenzene is in the cis form meaning that the cross-linking density of the material is reduced. The authors further showed that molecular motion of the rotaxane unit in the xerogel in air can provide macroscopic actuation properties in a pseudoreversible manner within a very short time scale ( 3 seconds) and that light energy can be transferred into mechanical energy, as exemplified by lifting a $174 \mathrm{mg}$ weight (Figure $7 \mathrm{~d}(\mathrm{iii})$ ). While limited in number, these examples highlight the 
potential of polymers and polymer networks based on $[n]$ rotaxanes to produce responsive materials with original advanced physical properties.

In the recent years, based on the seminal work by Sauvage and co-workers describing the first [c2]daisy chain rotaxane ${ }^{[115]}$ these mechanically interlocked molecules have emerged as a very promising molecular unit to construct muscle-like materials. ${ }^{[16]}$ In 2009 , the groups of Stoddart and Grubbs independently reported the first syntheses of short pH-responsive [c2]daisy chain polymers using the copper-catalyzed azide/alkyne Huisgen cycloaddition reaction as step-growth polymerization process. ${ }^{[117,118]}$ The synthetic macromolecules showed only low degree of polymerization (DP of 11 for Stoddart and 22 for Grubbs) as determined by GPC analyses, mainly because of solubility problems occurring during the polymerization of the [c2]daisy chain monomers. Nevertheless, both groups could demonstrate the possibility to extend their polymer chain upon $\mathrm{pH}$ modulation. The group of Stoddart even showed that the fully reversible extension/contraction movements can be cycled several times (up to 20) and that actuation of the polymer is indeed faster than the one of the monomer. ${ }^{[119]}$ However, the low polymerization degree of the macromolecules did not allow to draw conclusions on the possibility to amplify the molecular motion of [c2]daisy chains up to higher length scales. The first amplification of molecular motion across one length scale came up few years later when our group reported the metallo-supramolecular polymerization of $\mathrm{pH}$-responsive bistable [c2]daisy chain rotaxanes. ${ }^{[120]}$ The supramolecular approach allowed reaching polymers with high solubility in organic solvents and DP of $\sim 2900$ units, as determined by complementary light and neutron scattering experiments (DLS, SLS, and SANS). This set of experiment was of particular importance to demonstrate the single wormlike chain nature of the polymers, both in their extended and contracted states, and to determine that their experimental linear mass densities and contour lengths $\left(L_{c}\right)$ were in agreement with the dimensions expected from the DP multiplied by the size of each monomer. It provided strong evidences of an amplification of the mechanical actuation by an internal gliding of the 
polymer chains from the nanometric length scale $(\Delta l=1.2 \mathrm{~nm}$ for an individual [ $c 2$ ]daisy chain rotaxane) up to the micrometric scale $\left(\Delta L_{c}=6.5 \mu \mathrm{m}\right.$ for a full polymer chain). Following an identical supramolecular polymerization process, the group of Huang showed that [c2]daisy chain polymers made of pillar[5]arenes can be also actuated in a controlled manner by changing the solvent polarity. ${ }^{[121]}$ The large fibrillar aggregates formed by the polymers and observed by electron microscopy and dynamic light scattering experiments, were contracted by changing the solvent from highly polar (DMSO) to non-polar (chloroform), in agreement with the mechanical behavior of the monomer upon decreasing solvent polarity. In 2016, by copolymerizing via hydrogen bonding interactions a bistable [c2]daisy chain monomer presenting 2-aminopyridine stoppers with a complementary uracilterminated hydrophobic linker, our group showed that single wormlike chains can be further bundled into large micrometric aggregates which can change their morphologies upon $\mathrm{pH}$ stimulation as observed by TEM and AFM imaging experiments. ${ }^{[122]}$ While aggregation of the polymer chains results from lateral $\pi-\pi$ and van der Waals interactions between linkers, the change in morphologies can be explained by the actuation of the mechanical bonds which rigidifies the polymer chain, but also by its steric influence on the hydrogen-bond units when contracting the monomers, and that leads to a shortening of the polymer chains by partial depolymerization. All these achievements have paved the way towards the further developments described below and involving mechanically active self-standing materials, in which macroscopic physical properties can be tuned by actuation of the molecular machines at the nanometric scale.

In this direction, Harada and coworkers designed a material by covalent polymerization of a cyclodextrin/azobenzene-based [ $c 2$ ]daisy chain rotaxane with a tetraPEG polymer backbone, which offers several advantages such as high water solubility, low absorption at the wavelengths necessary to actuate the daisy chain, low interaction with the azobenzene unit and sufficient flexibility (Figure 8a). ${ }^{[123]}$ Upon irradiation at different wavelengths, the 
hydrogel could shrink and expand in a reversible manner due to the trans $\leftrightarrow$ cis isomerization of the azobenzene units (Figure 8a (ii-iii)). Light irradiation of a thin plate of the material in either its hydrogel or its xerogel states showed a bending towards the source of light, mainly because of a partial light penetration (Figure 8a $(i v-v))$. Importantly, while the hydrogel could be reversibly contracted and extended within several minutes $(\sim 180)$, only UV light could actuate the xerogel, albeit within times as short as 4 seconds. Pseudoreversibility of the process on the xerogel was achieved by sequentially shining light on each side of the material. By modifying the azobenzene unit by a stilbene, which presents improved photochemical properties (reaction rate, conversion, quantum yield), the same group subsequently develop a material that can be actuated reversibly within few seconds by two different UV wavelengths (A $(\lambda=350 \mathrm{~nm})$ and $\mathrm{C}(\lambda=280 \mathrm{~nm})$ ) both in the hydrogel and the xerogel states. ${ }^{[124]}$ Increasing the size of the tetraPEG polymer backbone had a beneficial effect on the contractile and mechanical properties of the hydrogel, as monitored by tensile test experiments (Figure $8 \mathrm{~b}(i-i i))$. Compared to their initial system, actuation was quasi-reversible for up to 15 times within a minute for the xerogel (Figure 8b (iii)), which also showed increased stiffness as demonstrated by its ability to lift a weight 15 times heavier than itself.

Following a strategy using click chemistry to build covalent polymer network of bistable [c2]daisy chains, our group designed a chemical gel which could be actuated in a reversible manner by using $\mathrm{pH}$ modulation (Figure 9a). ${ }^{[125]}$ By basifying piece of chemical gel in solution, a contraction down to $\sim 60 \%$ in volume of the material was monitored (Figure 9a (ii)). Reversible extension of the gel occurred by acidifying the solution, and the actuation process could be repeated several times (Figure 9a (iii)). Importantly, the macroscopic actuating properties were unambiguously associated with the molecular motion of the $[c 2]$ daisy chain units, as determined by both high-resolution magic angle spinning (HRMAS) NMR and by neutron scattering experiments revealing the change in linear mass densities of the polymer chains associated to their "telescopic" contraction (Figure 9a (iv)). As an alternative to the 
formation of covalent polymer networks, our group showed that physical reticulation of single polymer chains can be an effective strategy to build materials which macroscopic properties that are modulated by the actuation of the mechanically interlocked units. ${ }^{[126]}$ Bistable [c2]daisy chain supramolecular polymers built from the dimerization of their ureidopyrimidinone (UPy) stoppers leads to physical gels in their extended state, due to the stacking of the UPy dimers (Figure 9b). Upon deprotonation of the secondary ammonium station that leads to a contraction of the [c2]daisy chain unit, transition to a solution occurs. This gel-sol transition (which is reversible upon $\mathrm{pH}$ modulation), arises from a partial depolymerisation due to a lower dimerization constant of the UPy in the contracted state resulting from the closer proximity of the crown ether macrocycle, which decreases the association constant of physical reticulation nodes. ${ }^{[127]}$ Here again, important advanced experiments by SANS and SAXS related the macroscopic response of the materials directly with the mechanical actuations of the molecular machines.

Besides examples dealing with the covalent attachment of a single molecular motor to a single polymer chain, ${ }^{[101,102]}$ the development of self-standing polymeric materials incorporating artificial molecular motors has been very limited and conducted mostly by our group to date. ${ }^{[128-130]}$ In 2015 , we proposed the synthesis of chemical polymer gels incorporating lightdriven rotary motors as mechanically active reticulating nodes and which display unprecedented actuation properties. ${ }^{[128]}$ To construct such materials, functionalization of an enantiopure molecular motor ${ }^{[131]}$ was made on its upper part with azide-terminated poly(ethylene glycol) chains $\left(\mathrm{M}_{\mathrm{w}} \sim 5000\right.$ or $\left.10000 \mathrm{~g} \cdot \mathrm{mol}^{-1}\right)$, and on its lower part with alkyneterminated tri(ethylene glycol) units. This motor-polymer conjugate was further engaged in an azide/alkyne click reaction to produce cross-linked chemical gels (Figure 10a $(i)$ ). In their swollen state, e.g. 10 weight $\%$ in toluene, and upon irradiation at $365 \mathrm{~nm}$, a macroscopic contraction of the material was observed, which was shown to result from the nanoscopic rotary motion of the motors which are able to twist pairs of polymer chains. After light 
irradiation, the gel shrinks in an isotropic manner to a minimum size around $20 \%$ of its initial volume (Figure 10a (iv)). Interestingly, AFM imaging of the gel before and after irradiation showed an increased average size of the diameters of the micrometric pores with irradiation, while SAXS measurements showed an increased scattered intensity upon irradiation (Figure 10a (ii-iii)). These experimental observations suggest that, upon rotation of the motors, polymer chains create new entanglements in domains of high density, while leaving between them new empty spaces responsible for the unexpected increase of the materials porosity (and in contrast with what could be observed when shrinking a gel by using a variation of temperature for instance). This mechanical behavior at the nanometric level translates also into a particular macroscopic physical property, i.e. an increased shear modulus upon UV light irradiation, as observed by rheological measurements (Figure 10a $(v)$ ). ${ }^{[130]} \mathrm{We}$ demonstrated that several parameters influence the maximum mechanical efficiency of these materials such as: 1) the concentration of motor-polymer conjugate prior to chemical reticulation, and which should be close to the critical overlap concentration of the polymer chains $\left(c^{*}\right)$ to ensure a maximum number of active reticulation nodes and a minimum of inactive entanglements; and 2) presence of heterogeneities which leads to the presence of polymer strands that are inactive during the contraction process (Figure 10a (vi). Notably, this material presents unique properties in its actuation principle compared to all the previous examples described in the literature because it uses the unique capacity of the motor to constantly rotate and to increase the work it does on the polymer network. Here, the macroscopic actuation is the result of the cycling trajectories of motors at nanoscale, with a speed of the global contraction related to the frequency of the machines. A such, it allows to convert light energy into elastic energy which is stored in the polymer twists of the material (as a conformational battery); an energy that could be in principle further exploited for any purpose if coupled to appropriate chemical modules. 
This is with the objective to couple this first generation of active gels that we envisioned solutions to make their contraction reversible. However, because of the necessary unidirectional rotation of the molecular motor in this initial configuration, untwisting of the polymer chains is impossible without inverting its chirality. This inversion of chirality would be very demanding to achieve in materials, and it would not be of prime interest as it would not create a desirable hysteresis in the contraction / expansion process in order to not cancel the work done by the motor during the contraction. With these ideas in mind, we designed a dual-light responsive material which consists in a mixture of motor and "modulator" units, these latter operating as rotational elastic releasers at a wavelength different from the motor (Figure 10b $(i)) .{ }^{[129]}$ We chose a dithienylethene photoswitch as modulator and designed the material so that one modulator is always connected to molecular motors. The overall functioning principle of this material can be described as follows: 1) upon irradiation with UV light, modulators remain in their closed form, thus acting as "locked" units which can sustain the twisting of the polymer chains produced by the motors rotations and leading to a global contraction of the material (Figure 10b (ii)); and 2) upon irradiation with visible light, motors stop rotating and modulators switch to their open form, generating new freely rotating C-C single bonds that can untwist the polymer chains and expand the material (Figure 10b (iii)). Interestingly, low quantities of modulators (down to few percents) are sufficient to produce a reversibly contractile material, albeit with slower kinetics. It should be noted here that the entire system going back and forth by swelling and syneresis is not a switch in itself, because the twisting of the polymer chains and their untwisting do not follow the same pathway. As such, the motor-modulator gel can be considered as a fully motorized system. This property was demonstrated by the simultaneous use of UV and visible light irradiation at different intensities allowed fine-tuning of the work output at photostationary state by modulating the relative frequencies of the two light-responsive rotary units (Figure $10 \mathrm{~b}(i v)$ ). We believe that this approach which consists in motorizing a system in some points of a connected network, 
and of using other (switchable/reversible) nodes of this network to collect and transform the mechanical energy generated by the motor, has the potential to be implemented in many ways for the design of active materials capable of various energy transductions out of thermodynamic equilibrium. In addition, this general approach of braiding pairs of polymer chains can also be useful at microscale, and even at nanoscale with individual modules, in order to transform a rotary motion in a linear motion, and to make use of the tunable work produced by a single (or by a few) motor(s), for instance to manipulate small objects such as nanoparticles or even cells.

\section{Conclusion and perspectives}

This progress report shows that, over a recent and short period of time, synthetic chemists have succeeded in the first implementations of molecular machines in materials science. An interesting aspect provided by some of the intrinsic structural properties of materials is their capacity to organize molecular machines in space, both in terms of global orientation and of mechanical communication between them. In return, the molecular machines can confer these materials with stimuli-responsive properties. We have seen in each of the works described the importance of these organizing effects in 2D and 3D materials going from Langmuir layers to self-assembled systems and polymers. In the case of 2D systems, it has been shown that the mechanical actuation can be preserved even in densely packed situations. In 3D materials, most of the studies have been performed in relatively soft and mobile molecular systems when coupling molecular mechanical actuations (in vesicles, liquid crystals, gels). This is not surprising regarding the advantage of working in a medium of relatively high mobility to thermally actuate the machine, and to transfer its motion through and between other elements. We think that, however, investigations towards less mobile phases - such as in elastomers for instance - could be envisioned and would be of prime interest from both fundamental and applicative aspects. Even gearing effects that would be accessible by coupling motors (or 
motors and simple rotors) in the solid state could be used as a promising design. Another interesting direction related to mobility aspects would consist in playing with a combination of nano-mechanical actuations (provided by the machines) and of reversible chemical bonds maintaining the matrix of the materials. This was already approached in the case of supramolecular polymers and physical sol-gel transitions therefrom, but one can imagine coupling other supramolecular and dynamic covalent bonds to change the entire dynamics and topology of self-standing materials, or to induce non-linear mechano-chemical effects producing waves or oscillations by playing with coupled actuation-reaction-diffusion effects. Regarding the mechanisms at work in the already existing systems, we have also seen that, in some cases, the transduction of the mechanical signal involves simple polarity or electronic changes at the level of the machine, with a collective response of the material that is not directly generated by a nano-mechanical actuation (such as wettability or conductivity). Hence, in these cases, other types of stimuli-responsive units could proceed in principle by the same mechanisms and generate similar macroscopic responses, such as it could be the case with configurational photochromic switches. However, in a number of other cases, we have seen that the extraction and the transfer of the mechanical nano-actuation from the machine towards mechanical actuations at higher length scales are possible. This original aspect features a "hierarchical mechanics" which is particularly novel in terms of materials design, and which can give access in principle to new properties and functions.

Another feature to be explored further is the capacity of molecular motors to cycle motion and to progressively increase the work they release to their environment. As we have seen, only a very few number of examples use this possibility to actuate molecular machines out of thermodynamic equilibrium (Figure 8), although systems made of such motors would be able in principle to produce work at all scales with the potential to store and release energies, or to access truly active and autonomous materials (in a life-like approach). Here the mechanical systems become regulated by the trajectories and frequencies of the actuators and of other 
modules that can regulate them. The study of synchronization and desynchronization phenomena will be certainly instrumental in this direction, for instance by using molecular motors in Langmuir monolayers or in polymer gels to generate non-linear motions such as waves.

We have also shown that, up to now, all the artificial molecular motors used in materials have been driven by light, a source of energy very convenient for many applications. However, one can also envision coupling motors in adapted materials matrix when fueled by electrical energy, or even by chemical energy using information ratchet mechanisms to produce work (Figure $1 \mathrm{~b}(\mathrm{iii}))$. This would represent a particularly interesting direction to design mechanically active biomaterials which could function for instance as smart implants by using ATP as a source of energy in the body.

Finally, all these materials, to gain further in performance, and in particular in the case of macroscopic actuators, will benefit from coupling their molecular and supramolecular bottomup constructions with top-down technologies coming from chemical and physical engineering. In particular, by using electrospinning or $3 \mathrm{D}$ printing, one can expect to increase their hierarchical levels of organizations and their degree of orientation, to implement macroscopic ratchets and large hysteresis phenomena, and therefore to improve their mechanical performances in terms of directionality of actuation, workload, and autonomy.

\section{Acknowledgements}

The authors thank the European Commission's Horizon 2020 Programme as part of the MSCA-ITN project ArtMoMa under grant no. 860434, and of the FET-Open project MAGNIFY under grant no. 801378. They also thank the LabEx CSC, the CNRS, and the University of Strasbourg. C.C.C.-V. thanks the icFRC and Solvay Company for an excellence doctoral fellowship.

Received: ((will be filled in by the editorial staff))

Revised: ((will be filled in by the editorial staff)) Published online: ((will be filled in by the editorial staff)) 


\section{References}

[1] M. W. Urban, Stimuli-Responsive Materials, The Royal Society Of Chemistry, 2016.

[2] M. A. C. Stuart, W. T. S. Huck, J. Genzer, M. Müller, C. Ober, M. Stamm, G. B. Sukhorukov, I. Szleifer, V. V. Tsukruk, M. Urban, F. Winnik, S. Zauscher, I. Luzinov, S. Minko, Nat. Mater. 2010, 9, 101.

[3] Z. G. Wei, R. Sandstroröm, S. Miyazaki, J. Mater. Sci. 1998, 33, 3743.

[4] M. Behl, A. Lendlein, Mater. Today 2007, 10, 20.

[5] E. Smela, Adv. Mater. 2003, 15, 481.

[6] F. Ge, Y. Zhao, Adv. Funct. Mater. 2019, 1901890.

[7] H. Cui, Q. Zhao, Y. Wang, X. Du, Chem. - An Asian J. 2019, 14, 2369.

[8] L. Montero de Espinosa, W. Meesorn, D. Moatsou, C. Weder, Chem. Rev. 2017, 117, 12851.

[9] P. Cai, B. Hu, W. R. Leow, X. Wang, X. J. Loh, Y.-L. Wu, X. Chen, Adv. Mater. 2018, $30,1800572$.

[10] R. Merindol, A. Walther, Chem. Soc. Rev. 2017, 46, 5588.

[11] J.-P. Sauvage, Angew. Chem. Int. Ed. 2017, 56, 11080.

[12] J. F. Stoddart, Angew. Chem. Int. Ed. 2017, 56, 11094.

[13] B. L. Feringa, Angew. Chem. Int. Ed. 2017, 56, 11060.

[14] E. R. Kay, D. A. Leigh, F. Zerbetto, Angew. Chem. Int. Ed. 2007, 46, 72.

[15] C. J. Bruns, J. F. Stoddart, The Nature of the Mechanical Bond, John Wiley \& Sons, Inc., Hoboken, NJ, USA, 2016.

[16] S. Erbas-Cakmak, D. A. Leigh, C. T. McTernan, A. L. Nussbaumer, Chem. Rev. 2015, $115,10081$.

[17] A. Coskun, M. Banaszak, R. D. Astumian, J. F. Stoddart, B. A. Grzybowski, Chem. Soc. Rev. 2012, 41, 19.

[18] A. C. Fahrenbach, C. J. Bruns, D. Cao, J. F. Stoddart, Acc. Chem. Res. 2012, 45, 1581. 
[19] M.-M. Russew, S. Hecht, Adv. Mater. 2010, 22, 3348.

[20] S. Kassem, T. van Leeuwen, A. S. Lubbe, M. R. Wilson, B. L. Feringa, D. A. Leigh, Chem. Soc. Rev. 2017, 46, 2592.

[21] M. Baroncini, S. Silvi, A. Credi, Chem. Rev. 2019, DOI:10.1021/acs.chemrev.9b00291.

[22] R. D. Astumian, Science 1997, 276, 917.

[23] N. Koumura, R. W. J. Zijlstra, R. A. van Delden, N. Harada, B. L. Feringa, Nature 1999, 401, 152.

[24] M. R. Wilson, J. Solà, A. Carlone, S. M. Goldup, N. Lebrasseur, D. A. Leigh, Nature 2016, 534, 235.

[25] C. Pezzato, C. Cheng, J. F. Stoddart, R. D. Astumian, Chem. Soc. Rev. 2017, 46, 5491.

[26] C. L. Brown, U. Jonas, J. A. Preece, H. Ringsdorf, M. Seitz, J. F. Stoddart, Langmuir 2000, 16, 1924.

[27] M. Asakawa, M. Higuchi, G. Mattersteig, T. Nakamura, A. R. Pease, F. M. Raymo, T. Shimizu, J. F. Stoddart, Adv. Mater. 2000, 12, 1099.

[28] C. P. Collier, G. Mattersteig, E. W. Wong, Y. Luo, K. Beverly, J. Sampaio, F. M. Raymo, J. F. Stoddart, J. R. Heath, Science 2000, 289, 1172.

[29] Y.-H. Kim, S. S. Jang, Y. H. Jang, W. A. Goddard, Phys. Rev. Lett. 2005, 94, 156801.

[30] A. H. Flood, E. W. Wong, J. F. Stoddart, Chem. Phys. 2006, 324, 280.

[31] C. P. Collier, J. O. Jeppesen, Y. Luo, J. Perkins, E. W. Wong, J. R. Heath, J. F. Stoddart, J. Am. Chem. Soc. 2001, 123, 12632.

[32] Y. Luo, P. C. Collier, J. O. Jeppesen, K. A. Nielsen, E. Delonno, G. Ho, J. Perkins, H. R. Tseng, T. Yamamoto, J. F. Stoddart, J. R. Heath, ChemPhysChem 2002, 3, 519.

[33] J. E. Green, J. Wook Choi, A. Boukai, Y. Bunimovich, E. Johnston-Halperin, E. DeIonno, Y. Luo, B. A. Sheriff, K. Xu, Y. Shik Shin, H.-R. Tseng, J. F. Stoddart, J. R. Heath, Nature 2007, 445, 414.

[34] T. J. Huang, H.-R. Tseng, L. Sha, W. Lu, B. Brough, A. H. Flood, B.-D. Yu, P. C. 
Celestre, J. P. Chang, J. F. Stoddart, C.-M. Ho, Nano Lett. 2004, 4, 2065.

[35] I. C. Lee, C. W. Frank, T. Yamamoto, H.-R. Tseng, A. H. Flood, J. F. Stoddart, J. O. Jeppesen, Langmuir 2004, 20, 5809.

[36] S. S. Jang, Y. H. Jang, Y.-H. Kim, W. A. Goddard, J. W. Choi, J. R. Heath, B. W. Laursen, A. H. Flood, J. F. Stoddart, K. Nørgaard, T. Bjørnholm, J. Am. Chem. Soc. 2005, 127, 14804.

[37] E. DeIonno, H.-R. Tseng, D. D. Harvey, J. F. Stoddart, J. R. Heath, J. Phys. Chem. B 2006, 110,7609 .

[38] H. Kim, W. A. Goddard, S. S. Jang, W. R. Dichtel, J. R. Heath, J. F. Stoddart, J. Phys. Chem. A 2009, 113, 2136.

[39] Y. H. Jang, W. A. Goddard, J. Phys. Chem. C 2010, 114, 4611.

[40] P. M. Mendes, W. Lu, H.-R. Tseng, S. Shinder, T. Iijima, M. Miyaji, C. M. Knobler, J. F. Stoddart, J. Phys. Chem. B 2006, 110, 3845.

[41] X. Guo, Y. Zhou, M. Feng, Y. Xu, D. Zhang, H. Gao, Q. Fan, D. Zhu, Adv. Funct. Mater. 2007, 17, 763.

[42] M. Clemente-León, A. Credi, M. V. Martínez-Díaz, C. Mingotaud, J. F. Stoddart, Adv. Mater. 2006, 18, 1291.

[43] A. Coskun, J. M. Spruell, G. Barin, W. R. Dichtel, A. H. Flood, Y. Y. Botros, J. F. Stoddart, Chem. Soc. Rev. 2012, 41, 4827.

[44] H.-R. Tseng, D. Wu, N. X. Fang, X. Zhang, J. F. Stoddart, ChemPhysChem 2004, 5, 111.

[45] Y. B. Zheng, Y.-W. Yang, L. Jensen, L. Fang, B. K. Juluri, A. H. Flood, P. S. Weiss, J. F. Stoddart, T. J. Huang, Nano Lett. 2009, 9, 819.

[46] T. J. Huang, B. Brough, C.-M. Ho, Y. Liu, A. H. Flood, P. A. Bonvallet, H.-R. Tseng, J. F. Stoddart, M. Baller, S. Magonov, Appl. Phys. Lett. 2004, 85, 5391.

[47] Y. Liu, A. H. Flood, P. A. Bonvallet, S. A. Vignon, B. H. Northrop, H.-R. Tseng, J. O. 
Jeppesen, T. J. Huang, B. Brough, M. Baller, S. Magonov, S. D. Solares, W. A.

Goddard, C.-M. Ho, J. F. Stoddart, J. Am. Chem. Soc. 2005, 127, 9745.

[48] S. S. Jang, Y. H. Jang, Y.-H. Kim, W. A. Goddard, A. H. Flood, B. W. Laursen, H.-R. Tseng, J. F. Stoddart, J. O. Jeppesen, J. W. Choi, D. W. Steuerman, E. DeIonno, J. R. Heath, J. Am. Chem. Soc. 2005, 127, 1563.

[49] E. Katz, O. Lioubashevsky, I. Willner, J. Am. Chem. Soc. 2004, 126, 15520.

[50] J. Berna, D. A. Leigh, M. Lubomska, S. M. Mendoza, E. M. Perez, P. Rudolf, G. Teobaldi, F. Zerbetto, Nat. Mater. 2005, 4, 704.

[51] T. Heinrich, C. H.-H. Traulsen, M. Holzweber, S. Richter, V. Kunz, S. K. Kastner, S. O. Krabbenborg, J. Huskens, W. E. S. Unger, C. A. Schalley, J. Am. Chem. Soc. 2015, 137, 4382.

[52] M. M. Pollard, M. Lubomska, P. Rudolf, B. L. Feringa, Angew. Chem. Int. Ed. 2007, $46,1278$.

[53] G. T. Carroll, M. M. Pollard, R. van Delden, B. L. Feringa, Chem. Sci. 2010, 1, 97.

[54] J. Vachon, G. T. Carroll, M. M. Pollard, E. M. Mes, A. M. Brouwer, B. L. Feringa, Photochem. Photobiol. Sci. 2014, 13, 241.

[55] G. London, G. T. Carroll, B. L. Feringa, Org. Biomol. Chem. 2013, 11, 3477.

[56] G. London, K.-Y. Chen, G. T. Carroll, B. L. Feringa, Chem. Eur. J. 2013, 19, 10690.

[57] K.-Y. Chen, O. Ivashenko, G. T. Carroll, J. Robertus, J. C. M. Kistemaker, G. London, W. R. Browne, P. Rudolf, B. L. Feringa, J. Am. Chem. Soc. 2014, 136, 3219.

[58] M. R. Diehl, D. W. Steuerman, H.-R. Tseng, S. A. Vignon, A. Star, P. C. Celestre, J. F. Stoddart, J. R. Heath, ChemPhysChem 2003, 4, 1335.

[59] R. Klajn, L. Fang, A. Coskun, M. A. Olson, P. J. Wesson, J. F. Stoddart, B. A. Grzybowski, J. Am. Chem. Soc. 2009, 131, 4233.

[60] A. Coskun, P. J. Wesson, R. Klajn, A. Trabolsi, L. Fang, M. A. Olson, S. K. Dey, B. A. Grzybowski, J. F. Stoddart, J. Am. Chem. Soc. 2010, 132, 4310. 
[61] B. Long, K. Nikitin, D. Fitzmaurice, J. Am. Chem. Soc. 2003, 125, 15490.

[62] E. Lestini, K. Nikitin, J. K. Stolarczyk, D. Fitzmaurice, ChemPhysChem 2012, 13, 797.

[63] C. Jia, H. Li, J. Jiang, J. Wang, H. Chen, D. Cao, J. F. Stoddart, X. Guo, Adv. Mater. 2013, 25, 6752 .

[64] Z.-Q. Cao, Q. Miao, Q. Zhang, H. Li, D.-H. Qu, H. Tian, Chem. Commun. 2015, 51, 4973.

[65] Q. Zhang, S.-J. Rao, T. Xie, X. Li, T.-Y. Xu, D.-W. Li, D.-H. Qu, Y.-T. Long, H. Tian, Chem 2018, DOI:10.1016/j.chempr.2018.08.030.

[66] R. A. van Delden, M. K. J. ter Wiel, M. M. Pollard, J. Vicario, N. Koumura, B. L. Feringa, Nature 2005, 437, 1337.

[67] M. M. Pollard, M. K. J. ter Wiel, R. A. van Delden, J. Vicario, N. Koumura, C. R. van den Brom, A. Meetsma, B. L. Feringa, Chem. Eur. J. 2008, 14, 11610.

[68] N. Pairault, R. Barat, I. Tranoy-Opalinski, B. Renoux, M. Thomas, S. Papot, Comptes Rendus Chim. 2016, 19, 103.

[69] W. Chen, C. A. Glackin, M. A. Horwitz, J. I. Zink, Acc. Chem. Res. 2019, 52, 1531.

[70] T. D. Nguyen, H.-R. Tseng, P. C. Celestre, A. H. Flood, Y. Liu, J. F. Stoddart, J. I. Zink, Proc. Natl. Acad. Sci. U. S. A. 2005, 102, 10029.

[71] T. D. Nguyen, Y. Liu, S. Saha, K. C. F. Leung, J. F. Stoddart, J. I. Zink, J. Am. Chem. Soc. 2007, 129, 626.

[72] M. Wang, T. Chen, C. Ding, J. Fu, Chem. Commun. 2014, 50, 5068.

[73] D. Tarn, D. P. Ferris, J. C. Barnes, M. W. Ambrogio, J. F. Stoddart, J. I. Zink, Nanoscale 2014, 6, 3335.

[74] H. Yan, C. Teh, S. Sreejith, L. Zhu, A. Kwok, W. Fang, X. Ma, K. T. Nguyen, V. Korzh, Y. Zhao, Angew. Chem. Int. Ed. 2012, 51, 8373.

[75] M. Li, H. Yan, C. Teh, V. Korzh, Y. Zhao, Chem. Commun. 2014, 50, 9745.

[76] J. Kaleta, J. Chen, G. Bastien, M. Dračínský, M. Mašát, C. T. Rogers, B. L. Feringa, J. 
Michl, J. Am. Chem. Soc. 2017, 139, 10486.

[77] S.-Y. Hsueh, C.-T. Kuo, T.-W. Lu, C.-C. Lai, Y.-H. Liu, H.-F. Hsu, S.-M. Peng, C. Chen, S.-H. Chiu, Angew. Chem. Int. Ed. 2010, 49, 9170.

[78] R. Arumugaperumal, P. Raghunath, M.-C. Lin, W.-S. Chung, Chem. Mater. 2018, 30, 7221.

[79] Z.-Q. Cao, Y.-C. Wang, A.-H. Zou, G. London, Q. Zhang, C. Gao, D.-H. Qu, Chem. Commun. 2017, 53, 8683.

[80] D. J. van Dijken, J. Chen, M. C. A. Stuart, L. Hou, B. L. Feringa, J. Am. Chem. Soc. 2016, 138,660 .

[81] J. Chen, F. K.-C. Leung, M. C. A. Stuart, T. Kajitani, T. Fukushima, E. van der Giessen, B. L. Feringa, Nat. Chem. 2018, 10, 132.

[82] F. K.-C. Leung, T. van den Enk, T. Kajitani, J. Chen, M. C. A. Stuart, J. Kuipers, T. Fukushima, B. L. Feringa, J. Am. Chem. Soc. 2018, 140, 17724.

[83] F. K.-C. Leung, T. Kajitani, M. C. A. Stuart, T. Fukushima, B. L. Feringa, Angew. Chem. Int. Ed. 2019, 58, 10985.

[84] A. S. Lubbe, C. Böhmer, F. Tosi, W. Szymanski, B. L. Feringa, J. Org. Chem. 2018, 83, 11008.

[85] W. Danowski, T. van Leeuwen, S. Abdolahzadeh, D. Roke, W. R. Browne, S. J. Wezenberg, B. L. Feringa, Nat. Nanotech. 2019, 14, 488.

[86] P. Deria, J. E. Mondloch, O. Karagiaridi, W. Bury, J. T. Hupp, O. K. Farha, Chem. Soc. Rev. 2014, 43, 5896.

[87] E. D. Baranoff, J. Voignier, T. Yasuda, V. Heitz, J. P. Sauvage, T. Kato, Angew. Chem. Int. Ed. 2007, 46, 4680.

[88] E. D. Baranoff, J. Voignier, T. Yasuda, V. Heitz, J. P. Sauvage, T. Kato, Mol. Cryst. Liq. Cryst. 2009, 509, 165.

[89] M. Yoshio, T. Mukai, K. Kanie, M. Yoshizawa, H. Ohno, T. Kato, Adv. Mater. 2002, 
14,351 .

[90] I. Aprahamian, T. Yasuda, T. Ikeda, S. Saha, W. R. Dichtel, K. Isoda, T. Kato, J. F. Stoddart, Angew. Chem. Int. Ed. 2007, 46, 4675.

[91] T. Yasuda, K. Tanabe, T. Tsuji, K. K. Coti, I. Aprahamian, J. F. Stoddart, T. Kato, Chem. Commun. 2010, 46, 1224.

[92] H. He, E. M. Sevick, D. R. M. Williams, J. Chem. Phys. 2018, 148, DOI 10.1063/1.5022134.

[93] R. A. van Delden, N. Koumura, N. Harada, B. L. Feringa, Proc. Natl. Acad. Sci. 2002, $99,4945$.

[94] R. Eelkema, B. L. Feringa, Chem. - An Asian J. 2006, 1, 367.

[95] R. Eelkema, M. M. Pollard, J. Vicario, N. Katsonis, B. S. Ramon, C. W. M. Bastiaansen, D. J. Broer, B. L. Feringa, Nature 2006, 440, 163.

[96] R. Eelkema, M. M. Pollard, N. Katsonis, J. Vicario, D. J. Broer, B. L. Feringa, J. Am. Chem. Soc. 2006, 128, 14397.

[97] A. Kausar, H. Nagano, Y. Kuwahara, T. Ogata, S. Kurihara, Chem. Eur. J. 2011, 17, 508.

[98] A. Bosco, M. G. M. Jongejan, R. Eelkema, N. Katsonis, E. Lacaze, A. Ferrarini, B. L. Feringa, J. Am. Chem. Soc. 2008, 130, 14615.

[99] S. J. Aßhoff, S. Iamsaard, A. Bosco, J. J. L. M. Cornelissen, B. L. Feringa, N. Katsonis, Chem. Commun. 2013, 49, 4256.

[100] J. Sun, R. Lan, Y. Gao, M. Wang, W. Zhang, L. Wang, L. Zhang, Z. Yang, H. Yang, Adv. Sci. 2018, 5, 1700613.

[101] D. Pijper, B. L. Feringa, Angew. Chem. Int. Ed. 2007, 46, 3693.

[102] D. Pijper, M. G. M. Jongejan, A. Meetsma, B. L. Feringa, J. Am. Chem. Soc. 2008, 130, 4541.

[103] Q. Wu, P. M. Rauscher, X. Lang, R. J. Wojtecki, J. J. De Pablo, M. J. A. Hore, S. J. 
Rowan, Science 2017, 358, 1434.

[104] H. Xing, Z. Li, Z. L. Wu, F. Huang, Macromol. Rapid Commun. 2018, 39, 1700361.

[105] H. W. Gibson, M. C. Bheda, P. T. Engen, Prog. Polym. Sci. 1994, 19, 843.

[106] T. Takata, Bull. Chem. Soc. Jpn. 2018, 92, 409.

[107] A. Harada, Y. Takashima, M. Nakahata, Acc. Chem. Res. 2014, 47, 2128.

[108] R. Klajn, M. A. Olson, P. J. Wesson, L. Fang, A. Coskun, A. Trabolsi, S. Soh, J. F. Stoddart, B. A. Grzybowski, Nat. Chem. 2009, 1, 733.

[109] S. Dong, Y. Luo, X. Yan, B. Zheng, X. Ding, Y. Yu, Z. Ma, Q. Zhao, F. Huang, Angew. Chem. Int. Ed. 2011, 50, 1905.

[110] M. Nakahata, S. Mori, Y. Takashima, H. Yamaguchi, A. Harada, Chem 2016, 1, 766.

[111] X. Ge, Y. He, X. Liang, L. Wu, Y. Zhu, Z. Yang, M. Hu, T. Xu, Nat. Commun. 2018, 9, 1.

[112] W. Zhang, E. DeIonno, W. R. Dichtel, L. Fang, A. Trabolsi, J.-C. Olsen, D. Benítez, J. R. Heath, J. F. Stoddart, J. Mater. Chem. 2011, 21, 1487.

[113] Q. Lin, X. Hou, C. Ke, Angew. Chem. Int. Ed. 2017, 56, 4452.

[114] Y. Takashima, Y. Hayashi, M. Osaki, F. Kaneko, H. Yamaguchi, A. Harada, Macromolecules 2018, 51, 4688.

[115] M. C. Jiménez, C. Dietrich-Buchecker, J.-P. Sauvage, Angew. Chem. Int. Ed. 2000, 39, 3284.

[116] A. Goujon, E. Moulin, G. Fuks, N. Giuseppone, CCS Chem. 2019, 1, 83.

[117] L. Fang, M. Hmadeh, J. Wu, M. A. Olson, J. M. Spruell, A. Trabolsi, Y. W. Yang, M. Elhabiri, A. M. Albrecht-Gary, J. F. Stoddart, J. Am. Chem. Soc. 2009, 131, 7126.

[118] P. G. Clark, M. W. Day, R. H. Grubbs, J. Am. Chem. Soc. 2009, 131, 13631.

[119] M. Hmadeh, L. Fang, A. Trabolsi, M. Elhabiri, A.-M. Albrecht-Gary, J. F. Stoddart, J. Mater. Chem. 2010, 20, 3422.

[120] G. Du, E. Moulin, N. Jouault, E. Buhler, N. Giuseppone, Angew. Chem. Int. Ed. 2012, 
$51,12504$.

[121] L. Gao, Z. Zhang, B. Zheng, F. Huang, Polym. Chem. 2014, 5, 5734.

[122] A. Goujon, G. Du, E. Moulin, G. Fuks, M. Maaloum, E. Buhler, N. Giuseppone, Angew. Chem. Int. Ed. 2016, 55, 703.

[123] K. Iwaso, Y. Takashima, A. Harada, Nat. Chem. 2016, 8, 625.

[124] S. Ikejiri, Y. Takashima, M. Osaki, H. Yamaguchi, A. Harada, J. Am. Chem. Soc. 2018, $140,17308$.

[125] A. Goujon, T. Lang, G. Mariani, E. Moulin, G. Fuks, J. Raya, E. Buhler, N. Giuseppone, J. Am. Chem. Soc. 2017, 139, 14825.

[126] A. Goujon, G. Mariani, T. Lang, E. Moulin, M. Rawiso, E. Buhler, N. Giuseppone, J. Am. Chem. Soc. 2017, 139, 4923.

[127] G. Mariani, A. Goujon, E. Moulin, M. Rawiso, N. Giuseppone, E. Buhler, Nanoscale 2017, 9, 18456.

[128] Q. Li, G. Fuks, E. Moulin, M. Maaloum, M. Rawiso, I. Kulic, J. T. Foy, N. Giuseppone, Nat. Nanotech. 2015, 10, 161.

[129] J. T. Foy, Q. Li, A. Goujon, J.-R. Colard-Itté, G. Fuks, E. Moulin, O. Schiffmann, D. Dattler, D. P. Funeriu, N. Giuseppone, Nat. Nanotech. 2017, 12, 540.

[130] J.-R. Colard-Itté, Q. Li, D. Collin, G. Mariani, G. Fuks, E. Moulin, E. Buhler, N. Giuseppone, Nanoscale 2019, 11, 5197.

[131] Q. Li, J. T. Foy, J.-R. Colard-Itté, A. Goujon, D. Dattler, G. Fuks, E. Moulin, N. Giuseppone, Tetrahedron 2017, 73, 4874. 
a) (i)

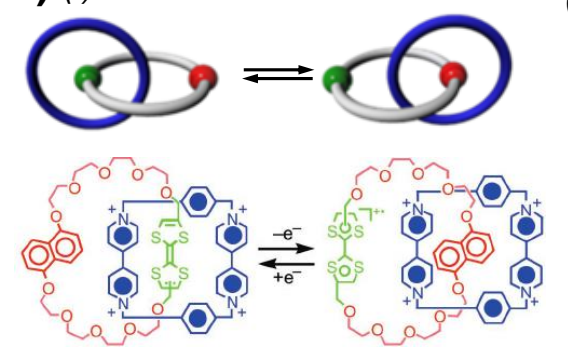

(ii)
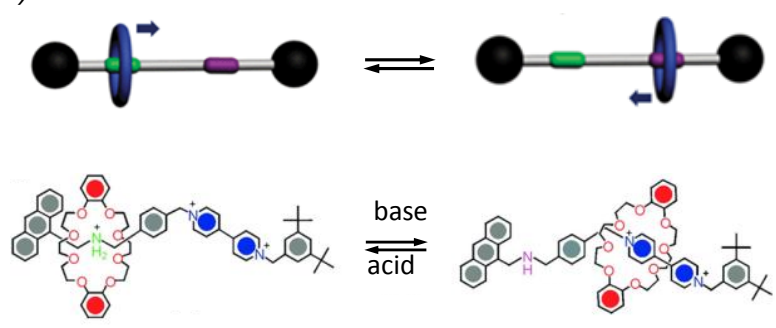
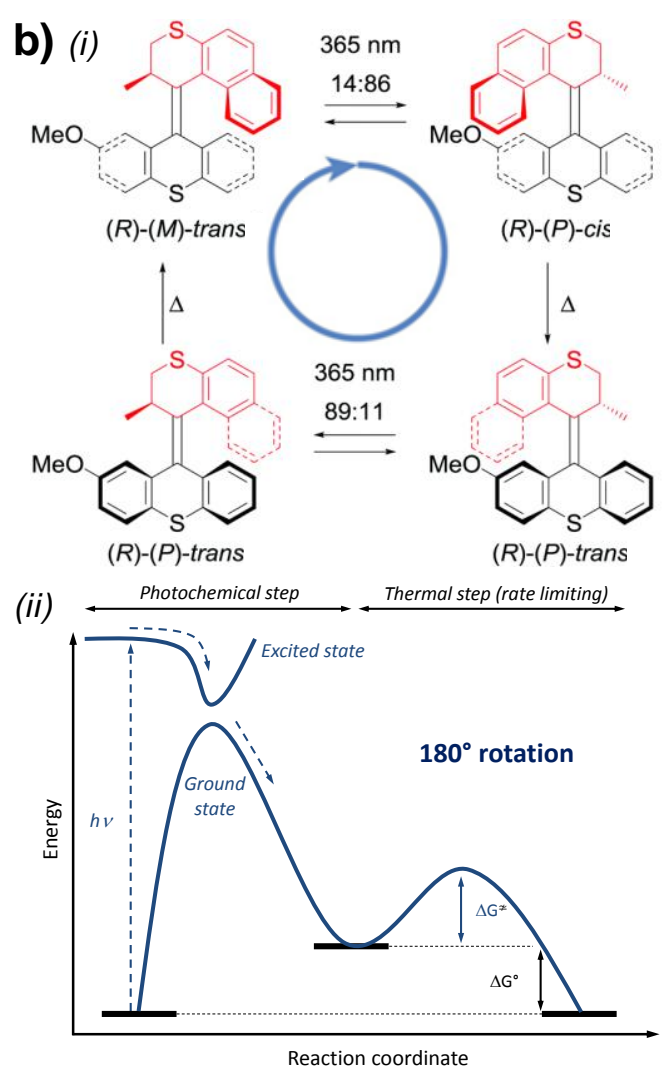

(iii)
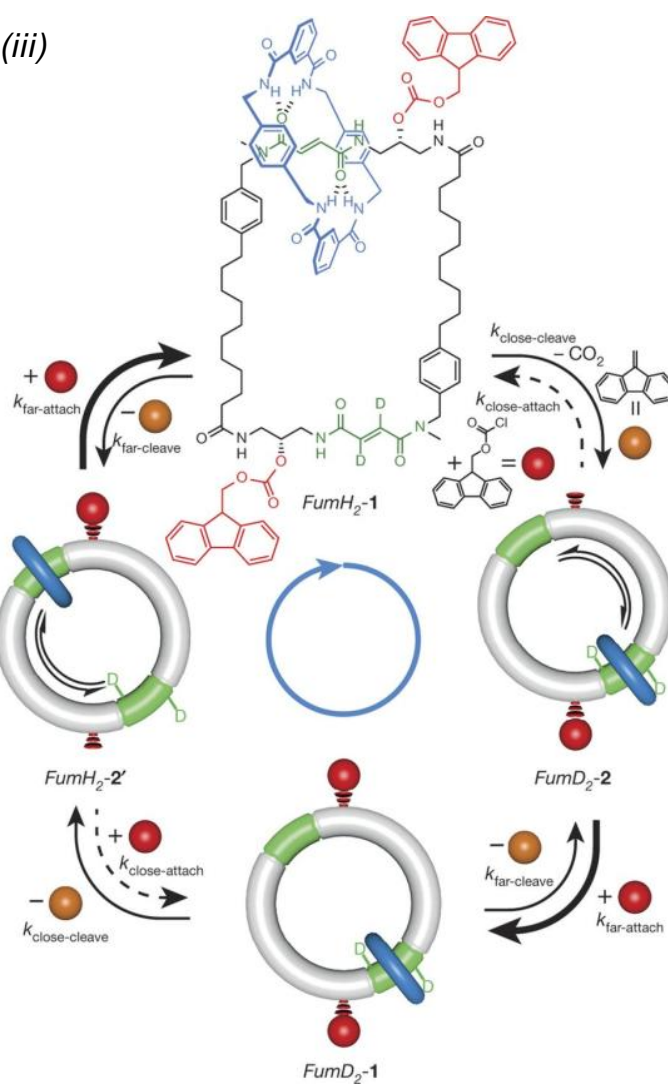

Figure 1. a) Schematic representations, and associated chemical structures, of a [2]catenane rotating by a red-ox process $(i)$, and of a [2] rotaxane shuttling by an acido-basic process (ii). The reversible switching motions between the stations, which take place over a typical length of $\approx 1 \mathrm{~nm}$, result from a change of the relative energies between the two possible states; b) (i) Typical mechanism for a unidirectional $360^{\circ}$ rotation of a light-driven molecular motor, based on power stroke actuation, combining two photochemical steps which isomerizes the double bond between cis and trans forms, and two thermal helix inversions which release the energy from unstable $(\mathrm{M})$ to stable $(\mathrm{P})$ isomers, thus enforcing a net clockwise rotation, (ii) General energy profile for a $180^{\circ}$ unidirectional rotation of a light-driven molecular motor such as in (i), and involving one photoisomerization step and one thermal helix inversion (THI). The activation energy of the THI determines the frequency of the motor, (iii) An autonomous chemically fueled catenane-based molecular motor based on an information ratchet mechanism. The clockwise rotation in this system is made possible because the Fmoc blocking group has a faster rate of attachment on the site far from the blue macrocycle (as compared to the nearest site), whereas the kinetics of cleavage is the same for both positions. Bold arrows indicate the major pathway of reaction, dashed arrows indicate the minor pathway and thin arrows indicate pathways that occur at similar rates. Adapted with permissions from references 17, 18, 20 and 28. 
a) (i)
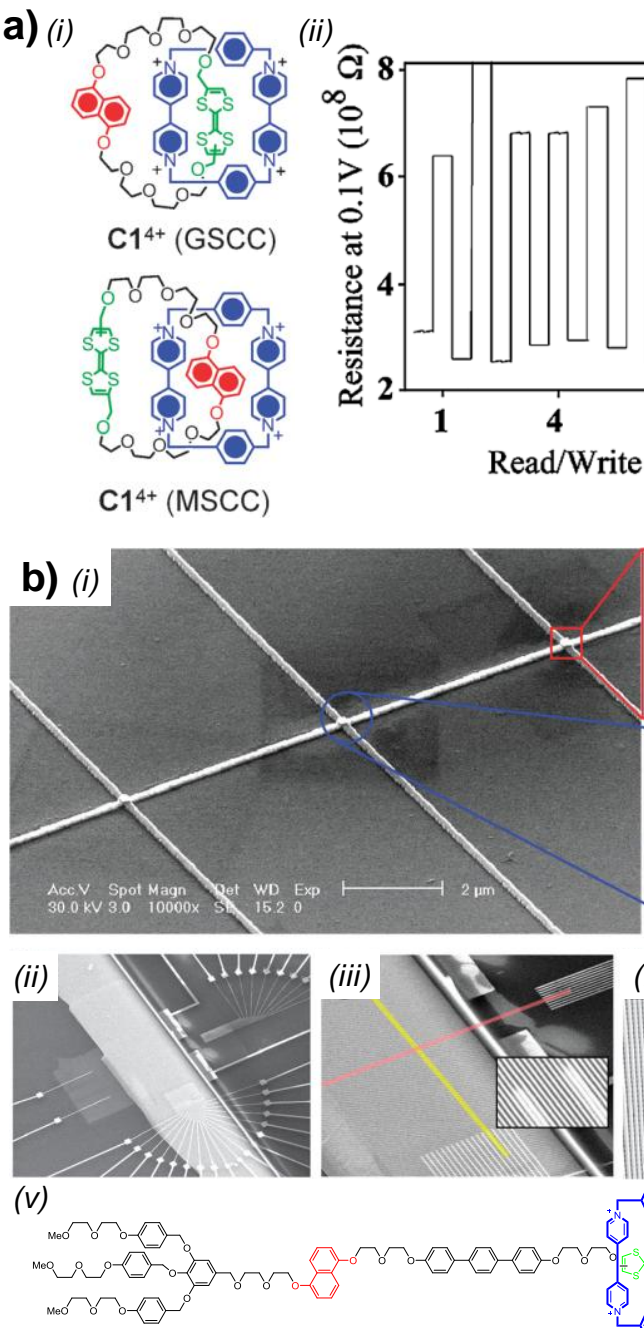

d)
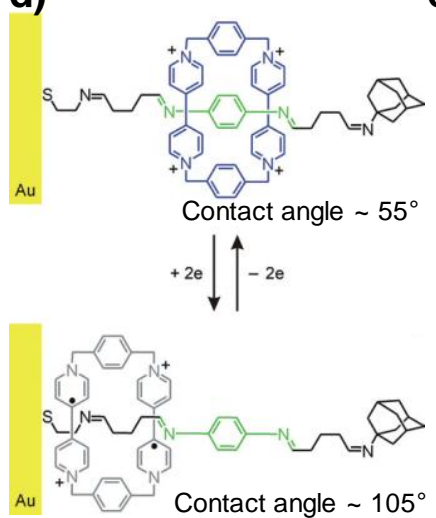
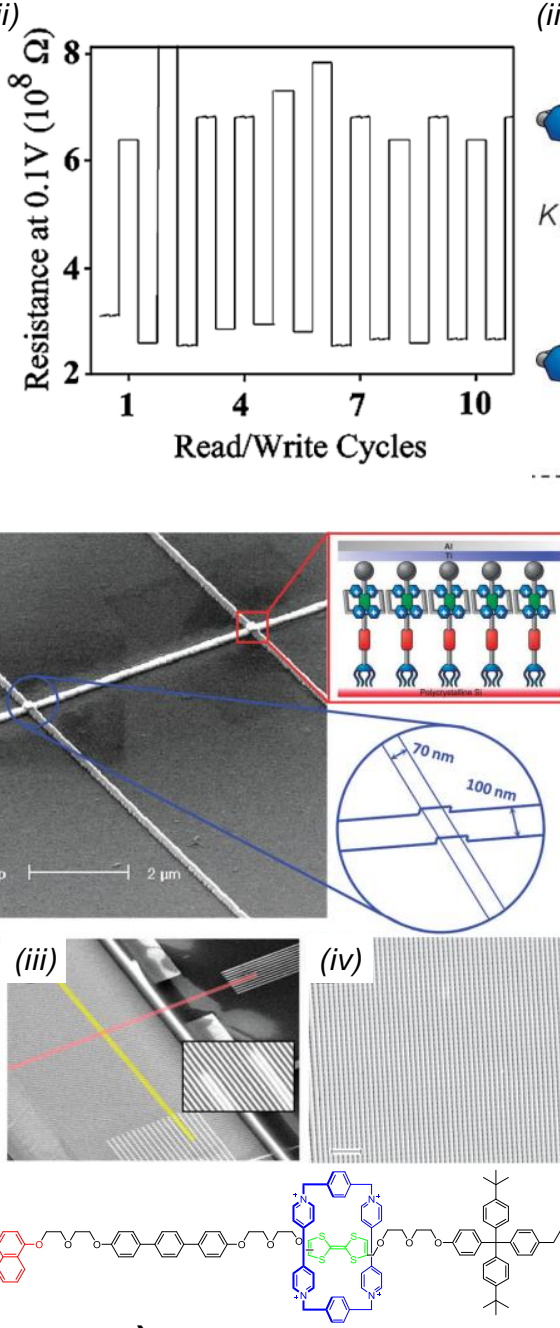

e)

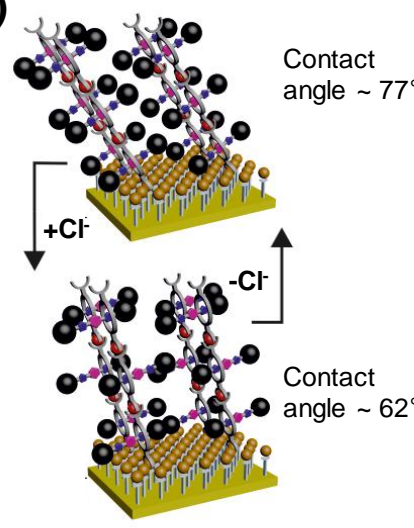

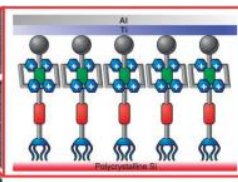

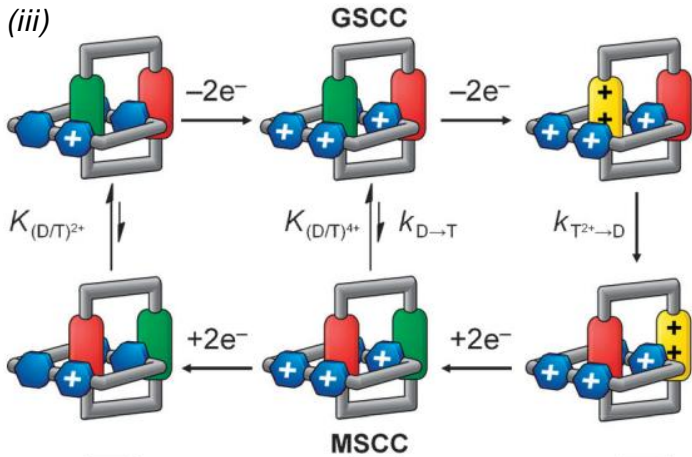

$2^{+}$

c) (i)

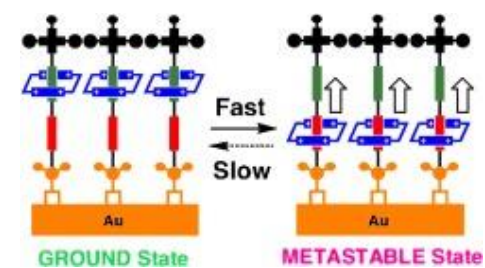

(ii)

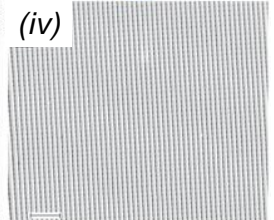

(iii)

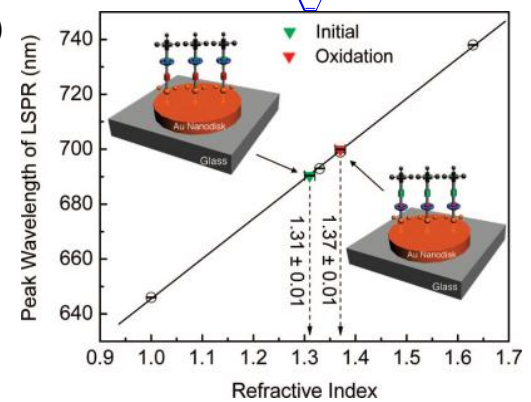

f)
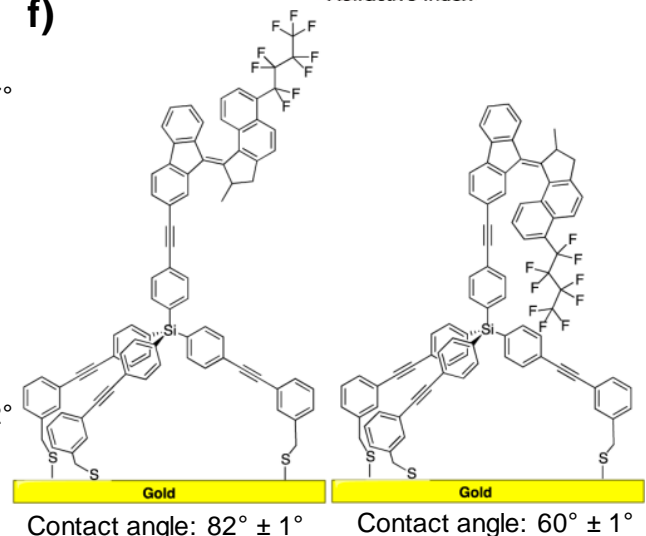

Figure 2. a) (i) Chemical structure of the ground-state co-conformer (GSCC) and the metastable-state co-conformer (MSCC) of the first [2]catenane $\mathbf{C 1}^{\mathbf{4 +}}$ used to build a molecular switch tunnel junction, (ii) device cycling performance for several on/off cycles, the resistance was measured at $0.1 \mathrm{~V}$, (iii) switching cycles for a bistable [2]catenane, the green, red, yellow and blue sites correspond to the tetrathiafulvalene (TTF), the 1,5-dioxynaphtalene (DNP), the $\mathrm{TTF}^{2+}$ dication and the CBPQT ring, respectively; b) SEM images of (i) a MSTJ consisting of bistable [2] rotaxanes sandwiched between a 70nm-wide poly-silicon electrode and a $100 \mathrm{~nm}$-wide titanium-aluminium one, (ii) the entire device, (iii) cross-point of top- (red) and bottom- (yellow) nanowire electrodes, and (iv) 2500 junctions of the crossbar circuit (scale bar : $200 \mathrm{~nm}$ ), (v) Chemical structure of the amphiphilic [2] rotaxane used within this device; c) (i) Redox switching of a surface-confined [2]rotaxane self-assembled as a 
monolayer on a gold surface, (ii) Chemical structure of the [2] rotaxane used within an active plasmonic device based on molecular machine, (iii) evolution of the LSPR peak wavelength and the corresponding refractive index of the device before and after chemical oxidation; d) Evolution of the contact angle value measured on an electrochemically active device made of a self-assembled monolayer of an imine-based [2]rotaxane; e) Evolution of the contact angle value measured on an anion-sensitive device made of a supramolecular multilayer of a [2]rotaxane; f) Evolution of the contact angle value measured on a gold surface decorated with a light-responsive self-assembled monolayer of a tripodal second-generation rotary motor. Adapted with permissions from references 20, 43, 44, 45, 49, 51 and 57.
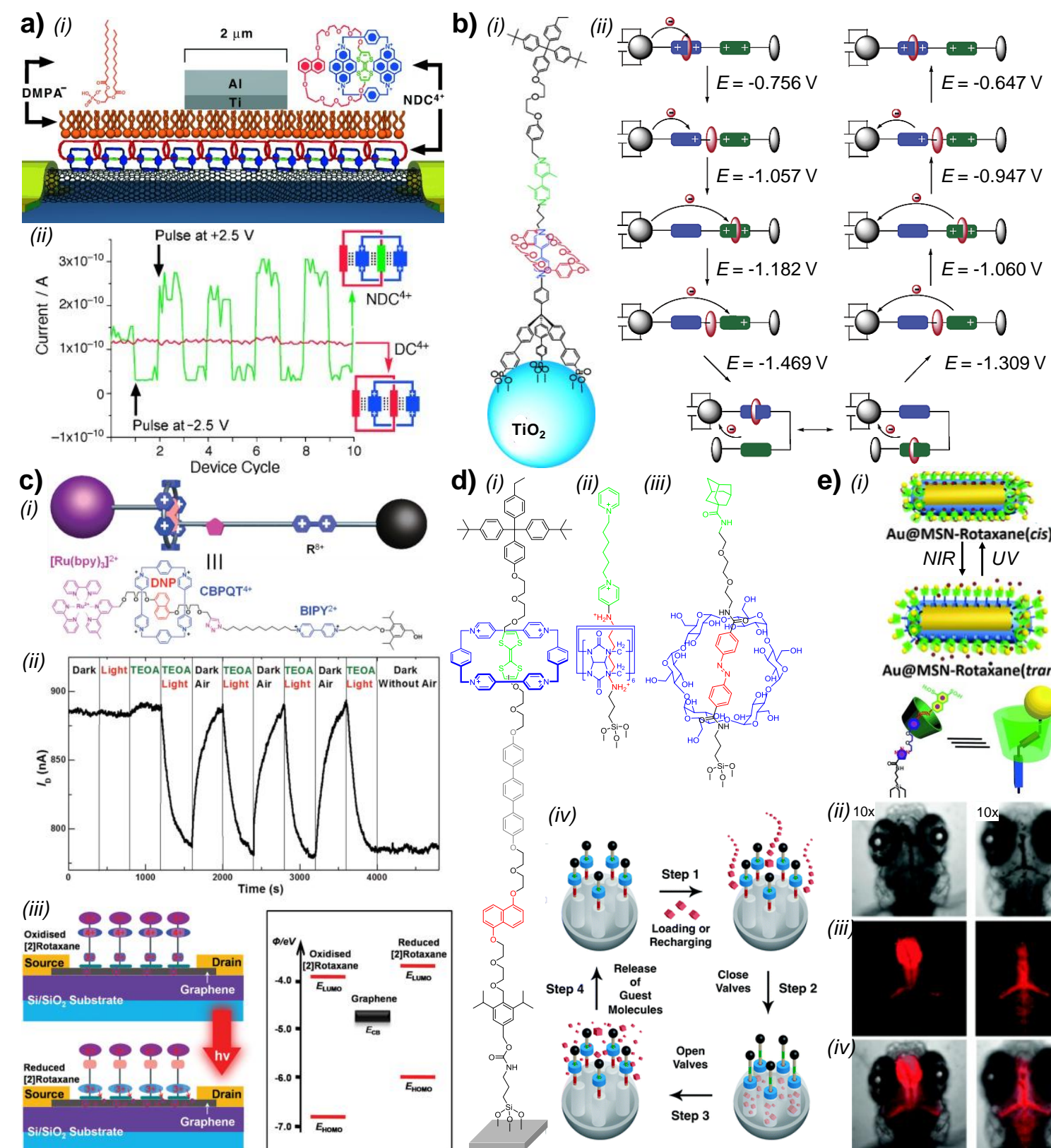

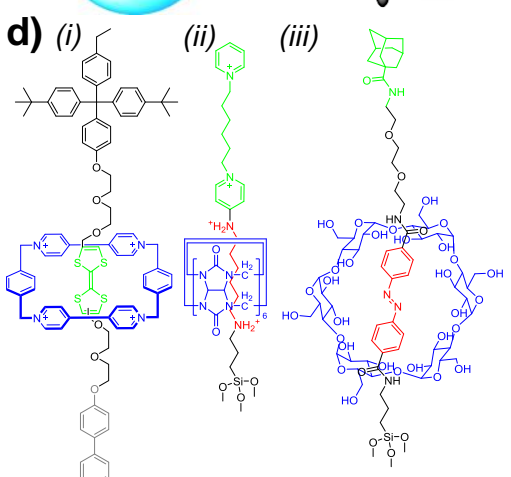

(iv)
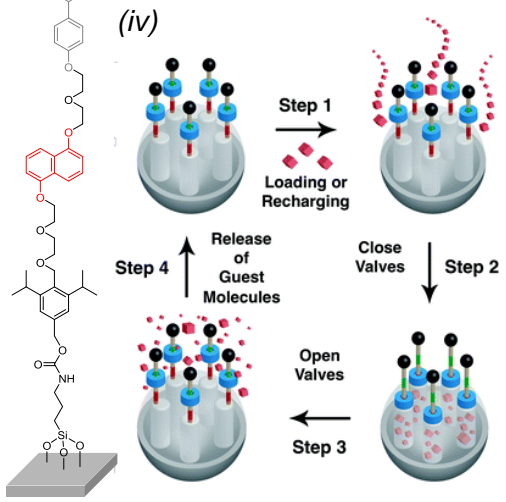

e) (i)

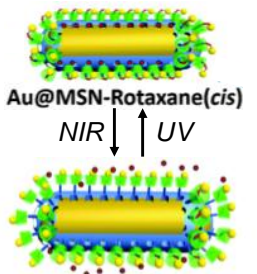

Au@MSN-Rotaxane(trans)

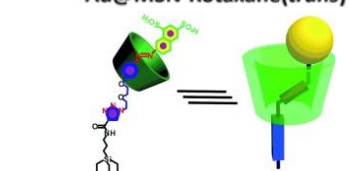

(ii) $10 \times$
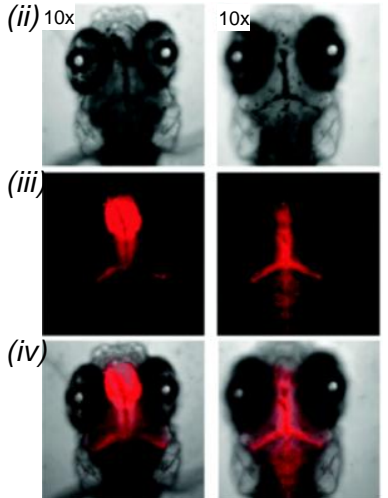

Figure 3. a) (i) Schematic representation of a MSTJ device built from carbon nanotubes and electroactive [2]catenanes, (ii) device cycling performance for this device (green), the red curve shows that the device made of the corresponding non-bistable [2]catenane has no cycling efficiency $\left(\mathrm{V}_{\text {read }}=100 \mathrm{mV}, \mathrm{V}_{\text {open }}=+2.5 \mathrm{~V}\right.$, and $\left.\mathrm{V}_{\text {close }}=-2.5 \mathrm{~V}\right)$; b) (i) Chemical structure of a tripodal redox-active [2] rotaxane mounted on $\mathrm{TiO}_{2}$ nanoparticles, (ii) schematic representation of the electrodynamics of a corresponding $\mathrm{TiO}_{2}$ film and the associated 
conformation change; c) (i) Chemical structure and schematic representation of the [2] rotaxane used to build a graphene transistor from molecular machines, (ii) cycling performance of this device upon different experimental conditions and oxidation/reduction cycles, TEOA: triethanolamine, (iii) graphical representation of the proposed photoswitching mechanism of this device and energy levels of the rotaxane/graphene interface; d) (i-iii) Chemical structures of the redox- $(i), \mathrm{pH}-($ ii) and light- (iii) responsive [2]rotaxanes used to build nanovalves, (iv) graphical representation of the functioning principle of nanovalves based on mesoporous nanoparticles and molecular machines; e) (i) Graphical representation of light-responsive mesoporous gold nanorods decorated with [2] rotaxanes, (ii-iv) Confocal images of the embryo heads of zebrafishes treated with the nanorods, right column: after irradiation with NIR for 1h, left column: without irradiation, (ii) bright field, (iii) Doxorubicin fluorescence, (iv) merged image. Adapted with permissions from references 58, 61, 63, 74 and 75 . 

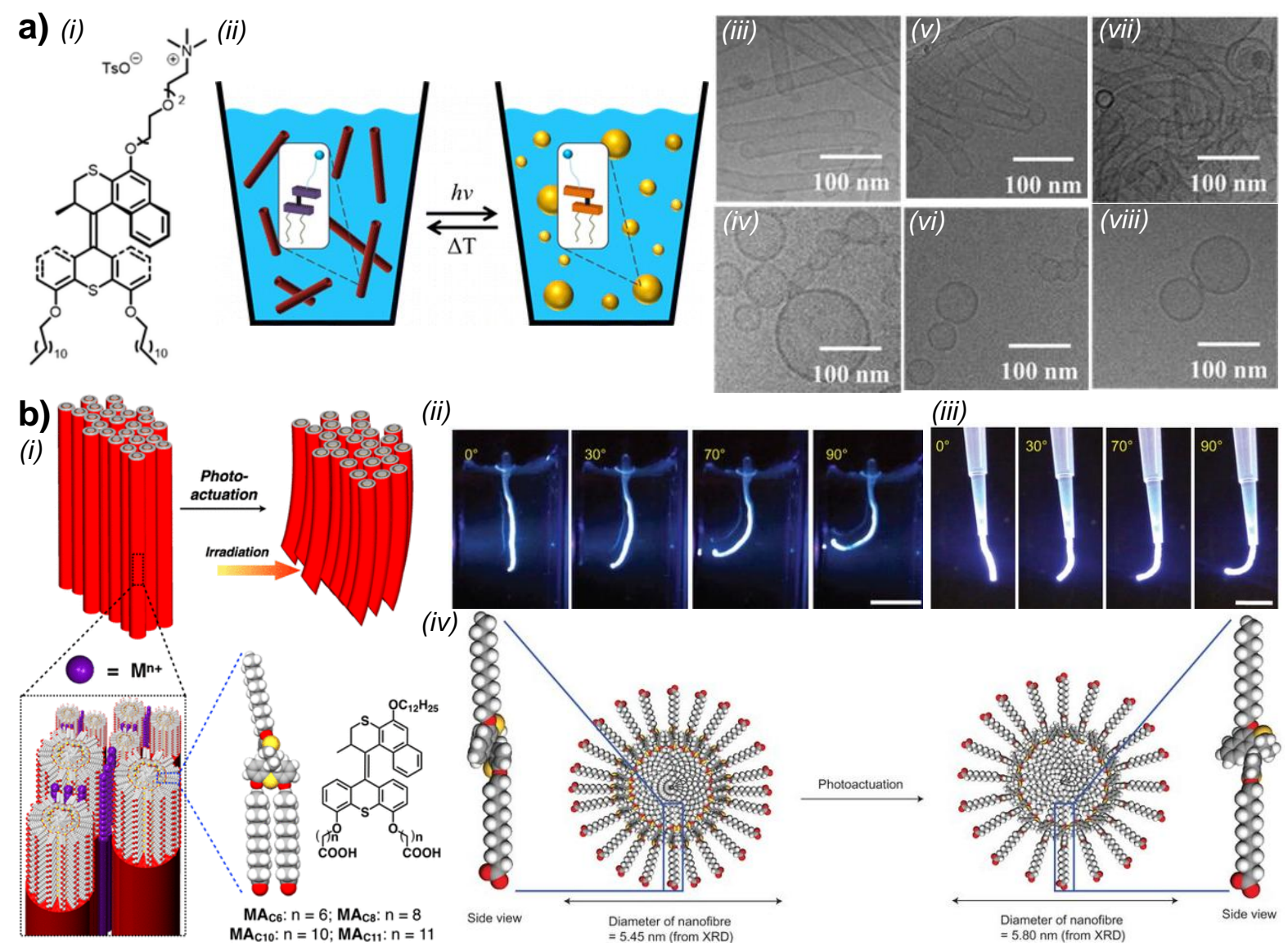

"
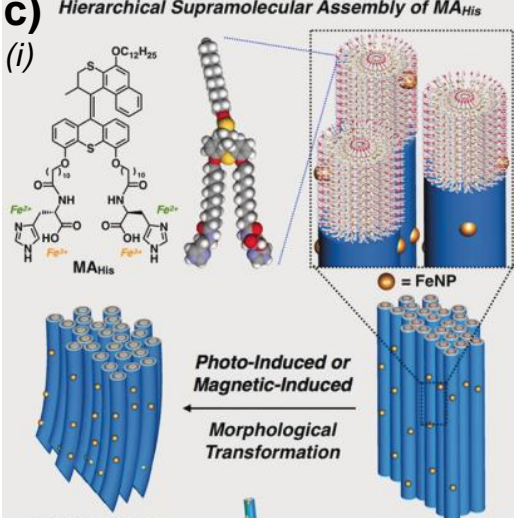

(ii)
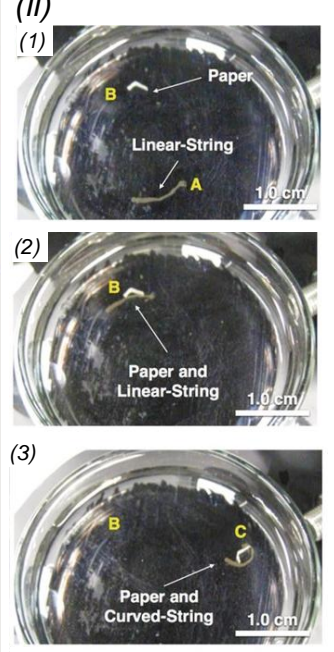

d) (i)

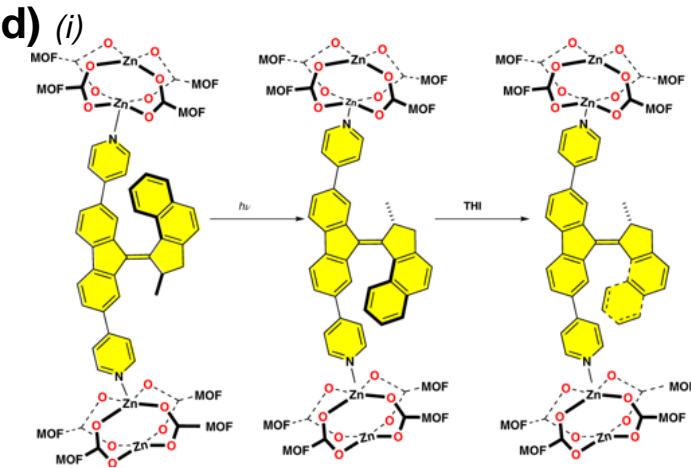

(ii)

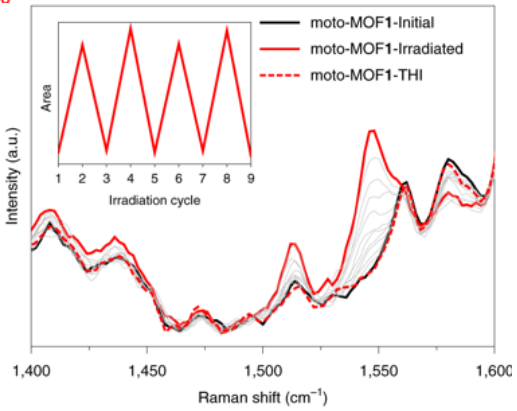

Figure 4. a) (i) Chemical structure of an amphiphilic second-generation rotary motor leading to the formation of (ii) self-assembled nanotubes, when mixed with DOPC (1,2-dioleoyl-snglycero-3-phosphocholine) in a 1:1 ratio in water, and which can be reversibly switched to vesicles upon light irradiation, (iii-viii) Cryo-TEM images of the nanostructures obtained after irradiation (10 $\mathrm{min}$ at $365 \mathrm{~nm}) /$ heating $\left(16 \mathrm{~h}\right.$ at $\left.50^{\circ} \mathrm{C}\right)$-freeze-thaw cycles ((iii): starting point, $(i v-v): 1^{\text {st }}$ cycle, (vi-vii): $2^{\text {nd }}$ cycle; (viii): irradiation); b) (i) Schematic representation of the hierarchical self-assembled structure made by the amphiphilic second-generation rotary motor with carboxylic acid end groups, (ii) Actuation in water of the corresponding supramolecular 
string over 60s irradiation from the left (scale bar: $0.5 \mathrm{~cm}$ ), (iii) Actuation in air of a macroscopic supramolecular string over $\sim 50 \mathrm{~s}$ UV irradiation from the left (scale bar: $0.5 \mathrm{~cm}$ ), (iv) Schematic representation of the evolution of the diameter of a nanofiber with light irradiation; c) (i) Chemical structure of a second-generation rotary motor decorated with histidine residues as end groups of the stator side chains and their hierarchical self-assembly upon addition of iron nanoparticles, schematic representation of their motion upon light or magnetic field actuation and their possible abilities to transport nanoscopic objects, (ii) Snapshots of a self-assembled string (position A, 1), its motion to position B (2), its light and magnet-induced motion to position C (3), and light-induced release of the paper (4); d) (i) Chemical structure of the rotary motor embedded in a Zn-MOF structure and its motion upon light irradiation and thermal helix inversion, (ii) Evolution of the Raman spectrum of the MOF upon irradiation at $395 \mathrm{~nm}$ and thermal isomerization at $20{ }^{\circ} \mathrm{C}$. Adapted with permissions from references $80,81,82,83$, and 85 .
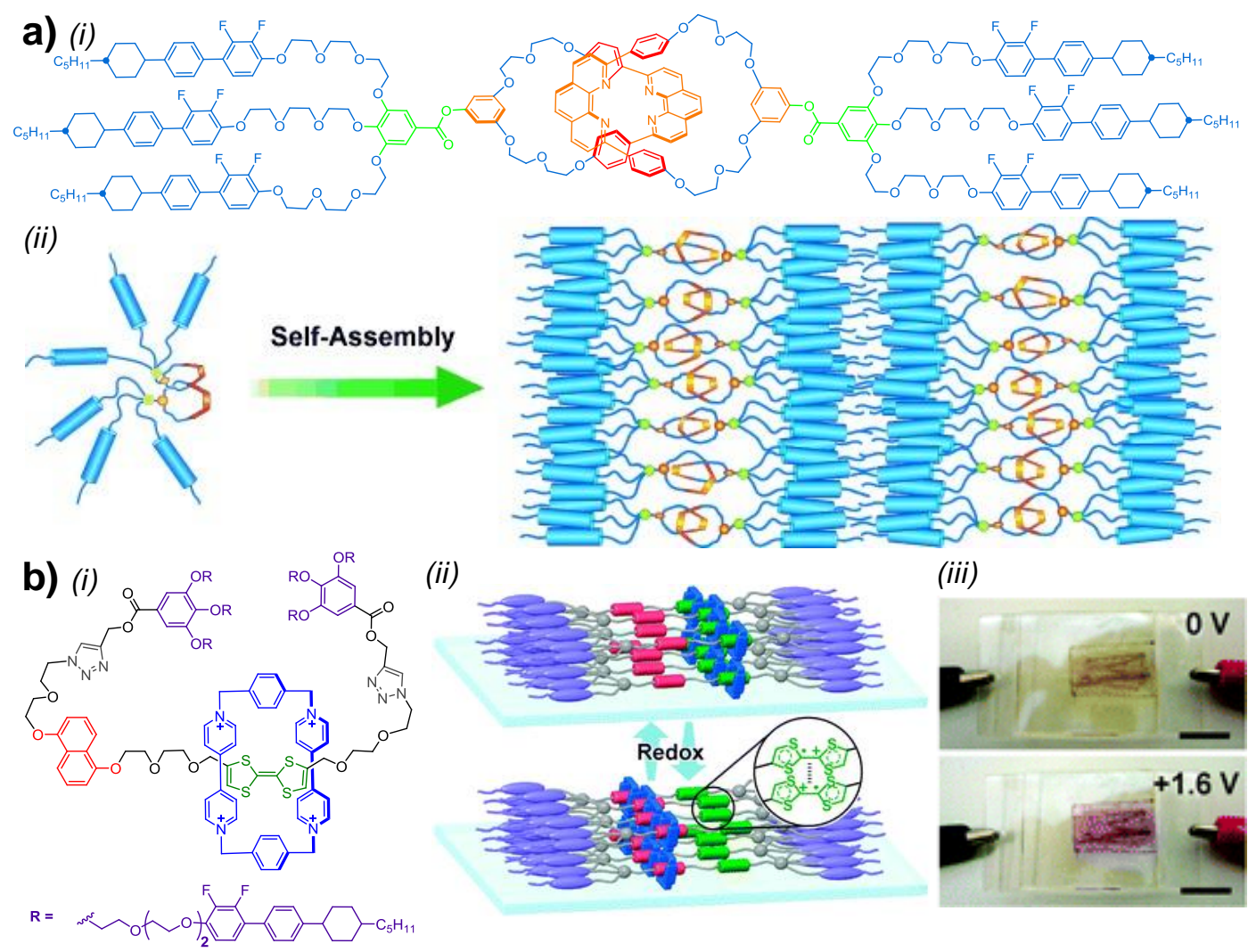

(ii)

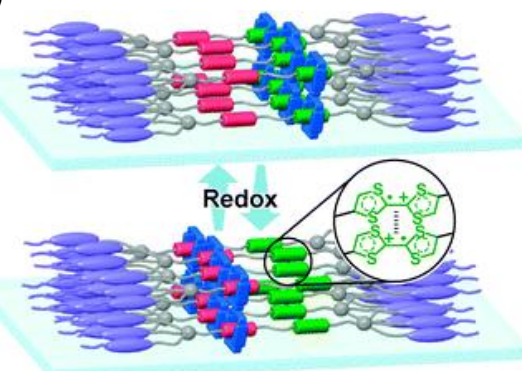

(iii)

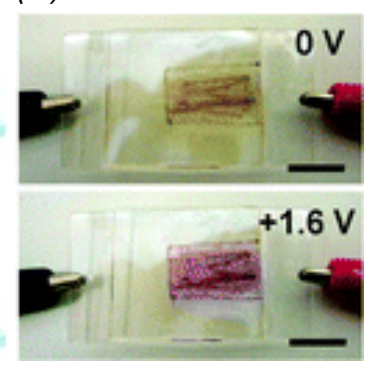

Figure 5. a) (i) Chemical structure of a liquid-crystalline [2]catenane and (ii) its plausible hierarchical self-assembly into a smectic A-type packing; b) (i) Chemical structure of a liquid-crystalline bistable [2] rotaxane, (ii) schematic representation of the corresponding film on an electrode surface and its actuation upon redox stimulation; (iii) images of an electrochemical cell made of this LC film after application of a positive bias. Adapted with permissions from references 87 and 91 . 
a)

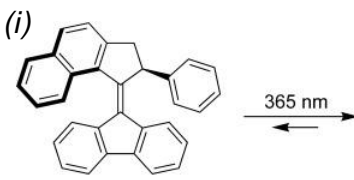

$(2 ' S)-(P)-1 \mathbf{a}$

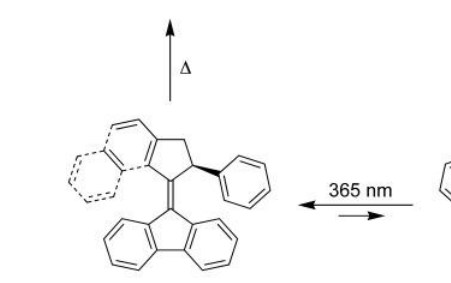

(ii)

(iv) LC Host E7

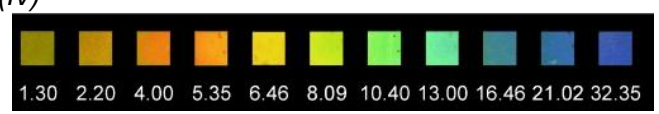

c) (i)

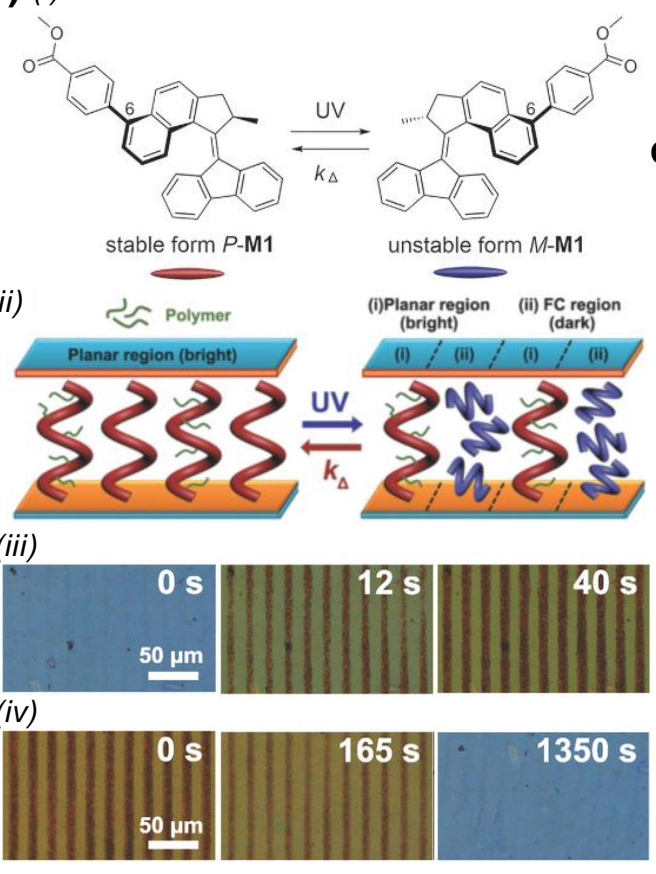

b)

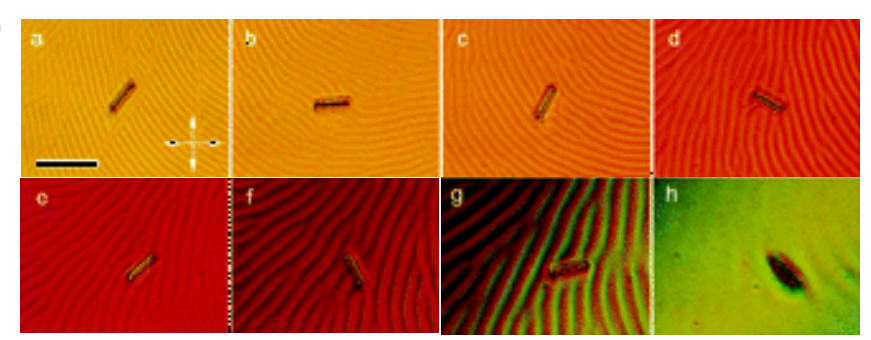

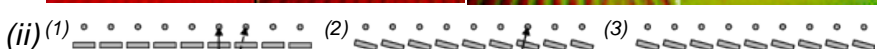

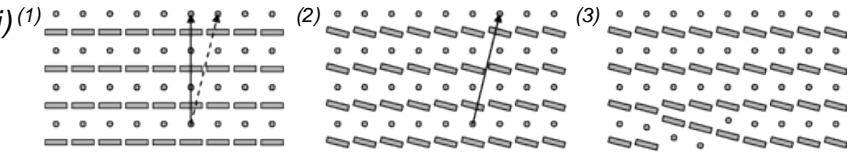

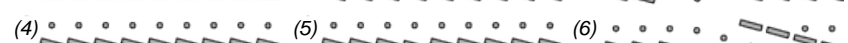
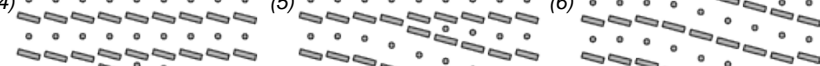

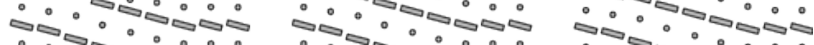

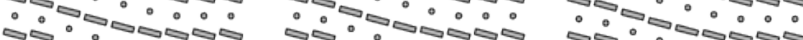

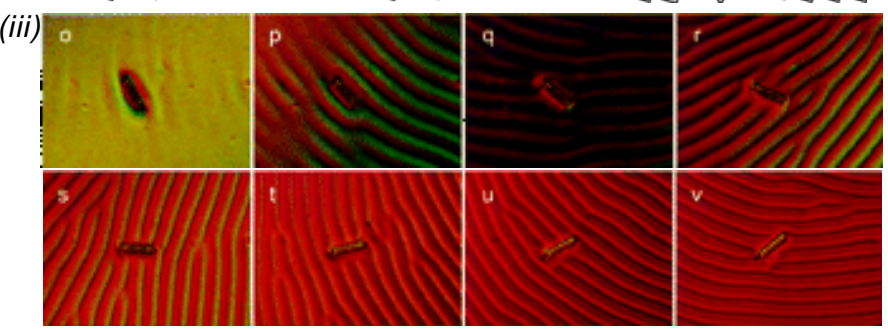

d) (i)
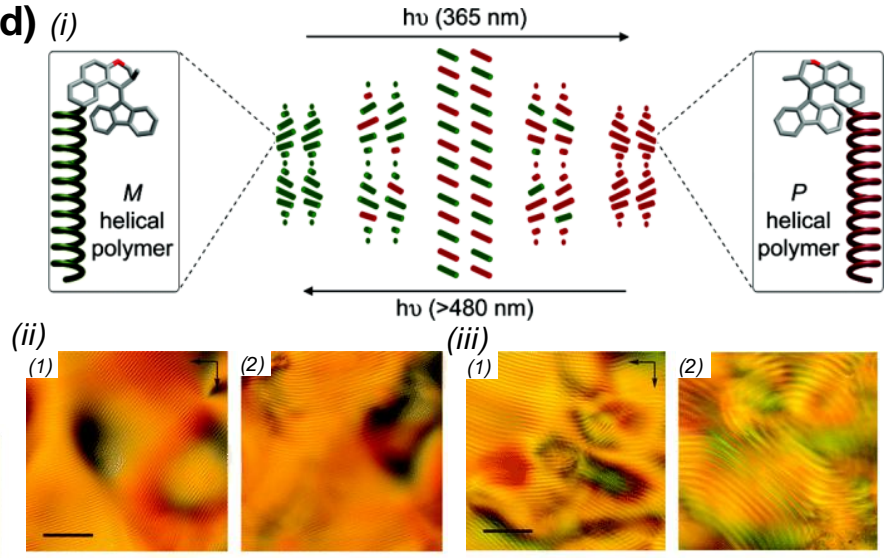

(iii)
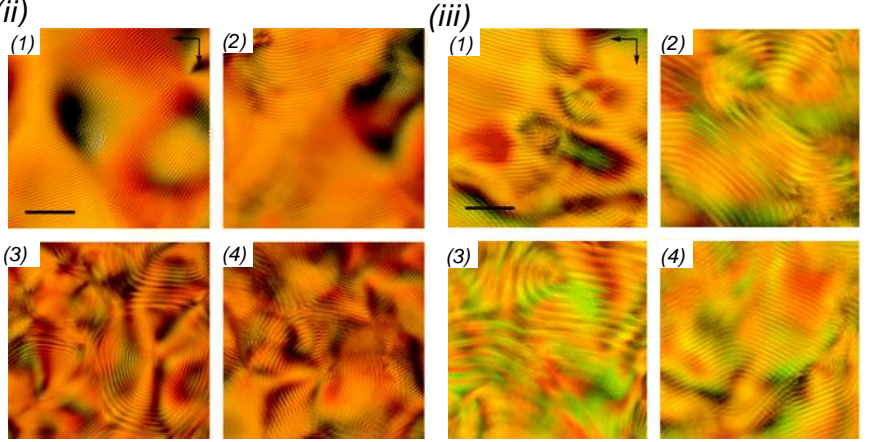

Figure 6. a) (i) Unidirectional rotary cycle of a fluorine-based motor, (ii) chemical structure of the mesogenic host E7 as a mixture of 4 compounds, (iii-iv) Evolution with time of the color of a film made of E7 doped with the fluorene-based motor (iii) upon UV irradiation $(365 \mathrm{~nm}$ ) and (iv) during thermal helix inversion; b) (i) Optical images of a $28 \mu \mathrm{m}$ glass rod rotating on a film made of E7 doped with the fluorene-based motor (panel a)) after irradiation with UV light (each image is taken at $15 \mathrm{~s}$ intervals, scale bar: $50 \mu \mathrm{m}$ ), (ii) Schematic representation of the mechanism proposed for the rotational reorganization of such LC film, (iii) Optical images of a $28 \mu \mathrm{m}$ glass rod rotating on a film made of E7 doped with the fluorene-based motor (panel a)) during thermal helix inversion ( $1^{\text {st }}$ image after $225 \mathrm{~s}$ at room temperature, each following image is taken at $15 \mathrm{~s}$ intervals, same scale as panel a)); c) (i) Chemical structure of a chiral rotary motor used to build surfaces with reversible dynamic LC diffraction grating properties, (ii) Schematic representation of the reversible dynamic polymer-stabilized cholesteric LC grating, (iii-iv) POM images of the corresponding grating during UV irradiation (iii) and after standing at room temperature (iv); d) (i) Schematic 
representation of the evolution (magnitude and sign of the helical pitch) with light irradiation of a cholesteric LC phase made of a polyisocyanate (PHIC) polymer decorated at one end with a molecular motor, (ii) Optical images of a film made of the PHIC polymer in toluene upon irradiation with UV light ((1): no irradiation, (2): $15 \mathrm{~min},(3)$ : $90 \mathrm{~min},(4)$ : $150 \mathrm{~min}$, scale bar: $50 \mu \mathrm{m}$ ), (iii) Optical images of a film made of a large excess of $(P)$ helical PHIC polymer in toluene upon irradiation with visible light at $>480 \mathrm{~nm}((1): 5 \mathrm{~h},(2): 6 \mathrm{~h},(3): 8 \mathrm{~h}$, (4): $10 \mathrm{~h}$, scale bar: $50 \mu \mathrm{m})$. Adapted with permissions from references 94, 96, 100 and 102 .

a) (i)

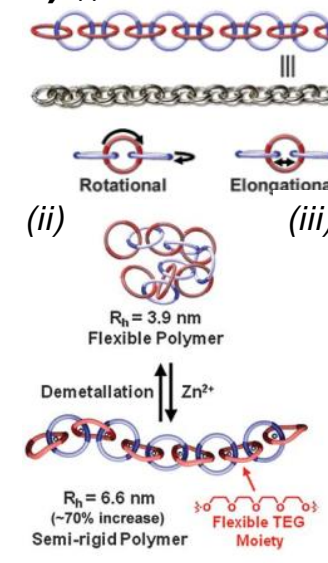

c) (i)

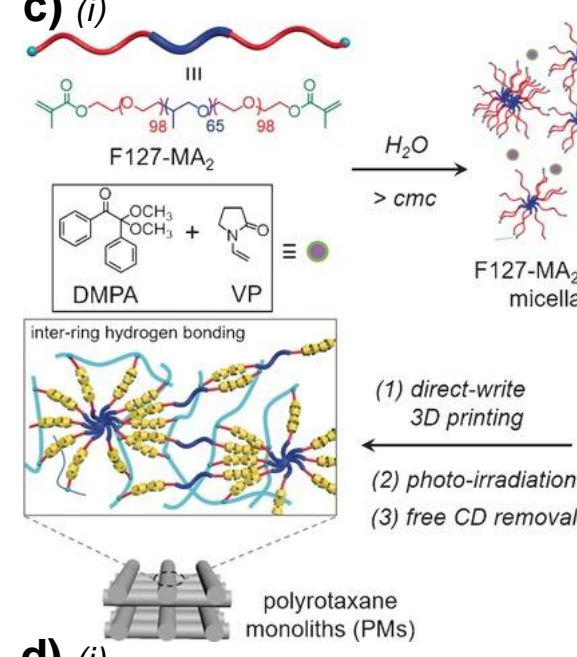

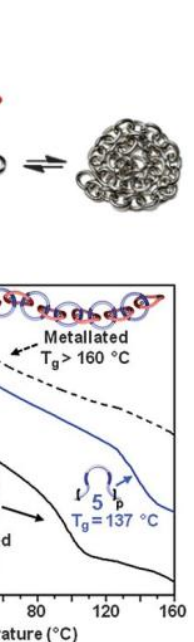
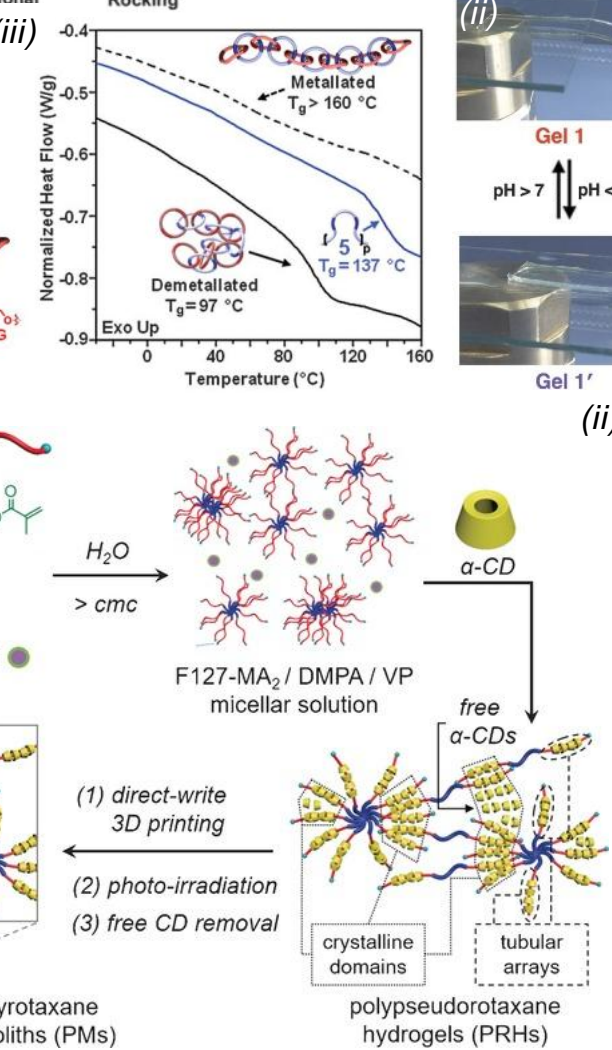

(ii) (1)

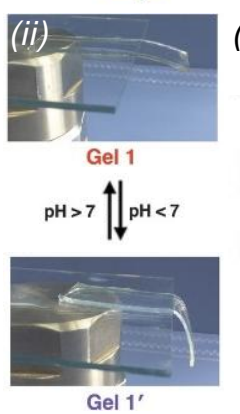

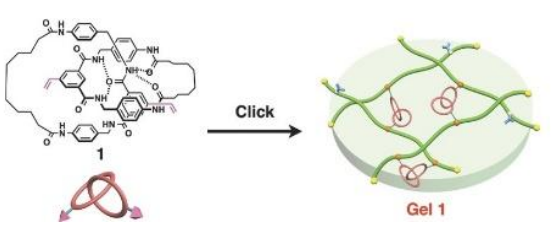

(iii)
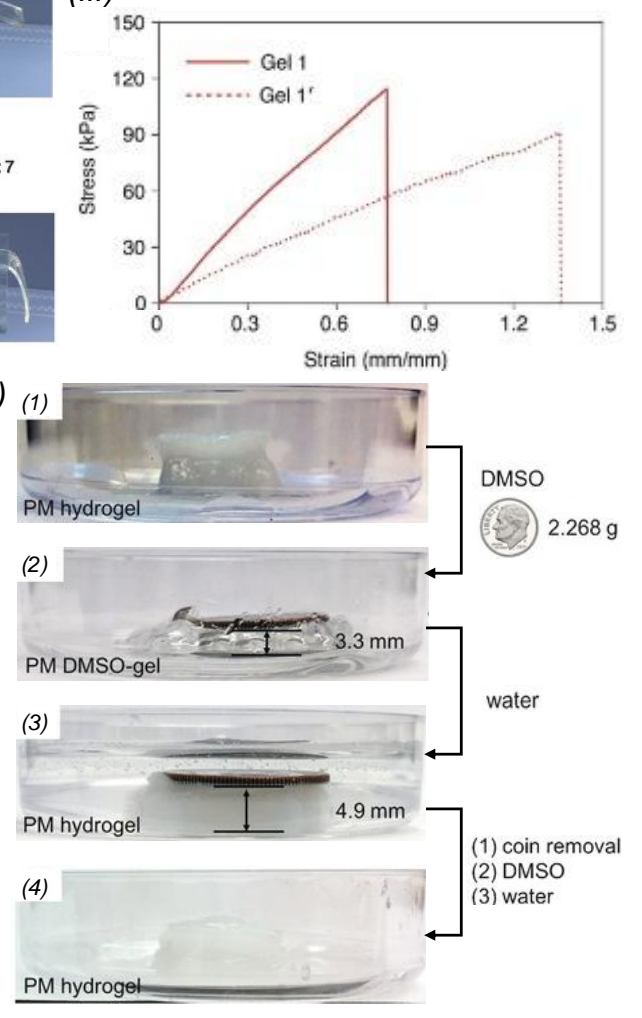

(ii)
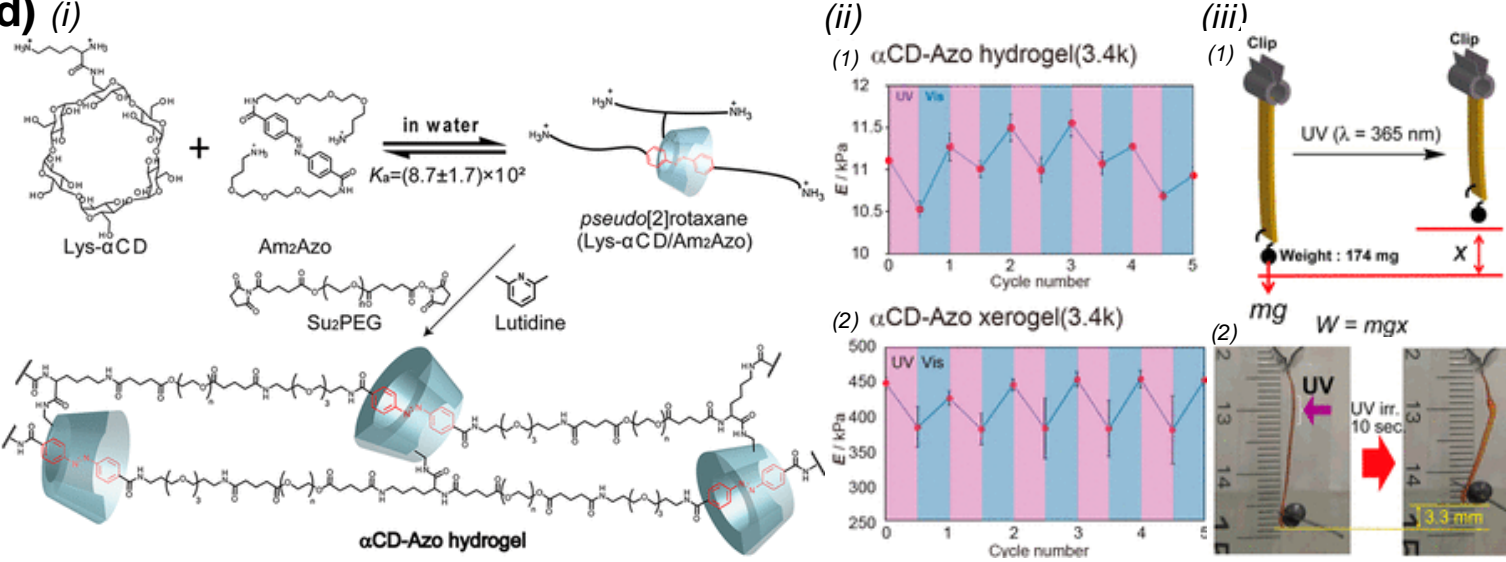

$m g \quad w=m g x$

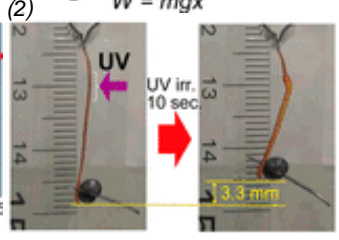

Figure 7. a) (i) Schematic representation of a poly $[n]$ catenane and the possible conformation motion of the rings, (ii) Reversible evolution of the hydrodynamic radius of the polycatenane 
leading to an evolution from flexible to semi-rigid structures upon metalation, (iii) Evolution of the glass transition temperature $\left(\mathrm{T}_{\mathrm{g}}\right)$ for the metallated and demetallated polymers as measured by DSC analysis; b) (i) Chemical structures of the polymer and catenane used to form a chemical gel with catenane units as cross-links, (ii) Evolution of a thin layer of gel upon $\mathrm{pH}$ modulation, (iii) Tensile stress-strain measurements on the gels obtained by modulating the $\mathrm{pH}$; c) (i) General principle used to build polyrotaxane monoliths by the direct-writing technique from PEO-PPO-PEO triblock copolymer $\left(\mathrm{F} 127-\mathrm{MA}_{2}\right)$ and $\alpha-$ cyclodextrin, (ii) Photographs of the corresponding hydrogel (1), its actuation by modulating the solvent used to swell it (2-3), and its size and shape recovery (4); d) (i) Covalent polymer network built from a pseudo[2]rotaxane and linear PEG units, (ii) Evolution of the Young modulus of the corresponding hydrogel (1) and xerogel (2) upon UV and Visible light irradiation over 5 cycles, (iii) (1) Schematic representation of the lift weight experiment upon UV irradiation and (2) corresponding images. Adapted with permissions from references 103, 104, 113 and 114.
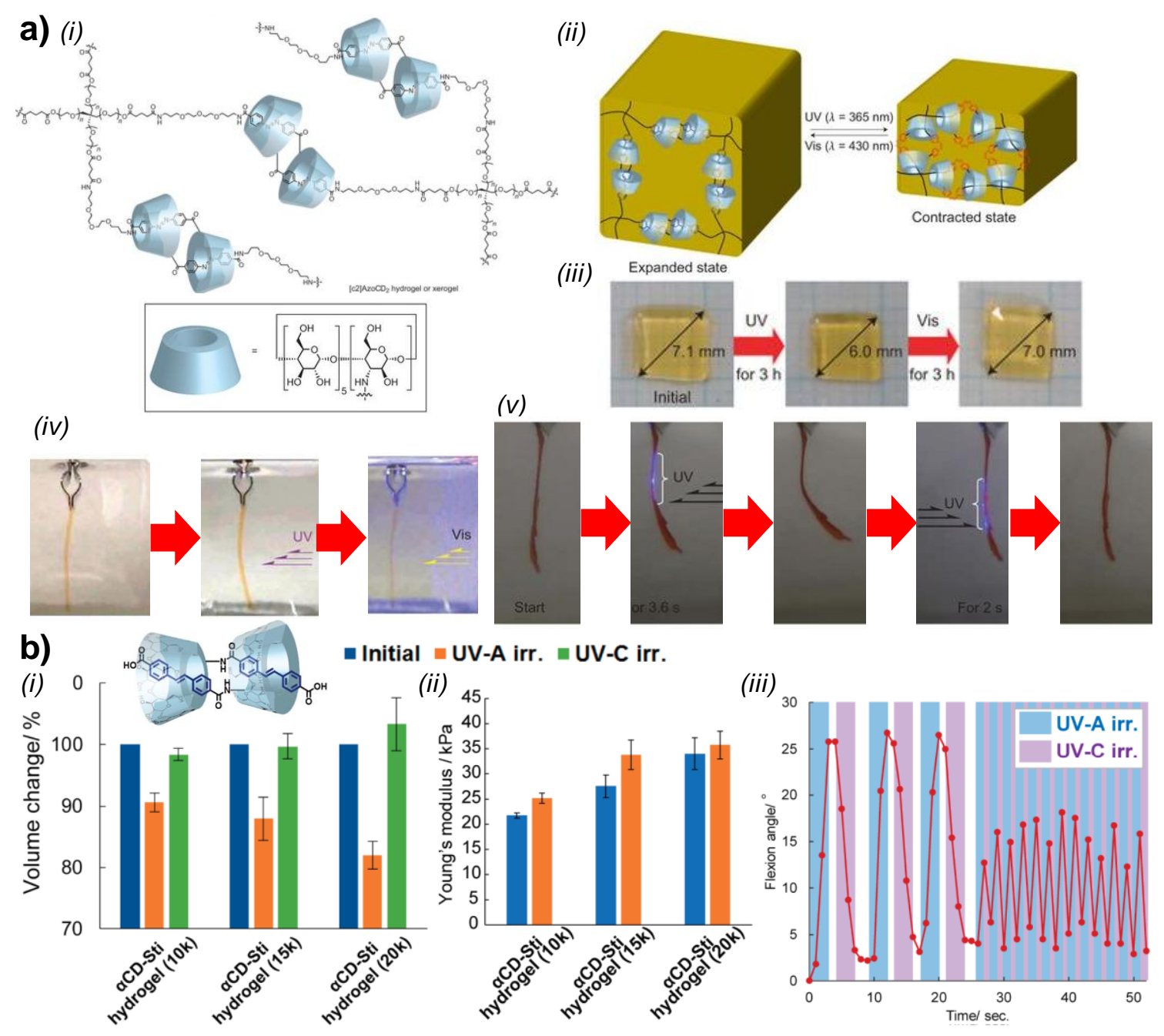

Figure 8. a) (i) Chemical structure of the covalent polymer network made of $\alpha$-cyclodextrinbased [c2]daisy chain rotaxanes, (ii) Schematic representation of the contraction and extension of a piece of gel upon UV and Visible light irradiation and (iii) photographs of the corresponding motion, (iv) Images of the motion of a thin plate of hydrogel upon UV irradiation $(365 \mathrm{~nm})$ for $180 \mathrm{~min}$ and then visible light irradiation $(480 \mathrm{~nm})$ for $180 \mathrm{~min},(v)$ Images of the motion of a thin plate of xerogel irradiated sequentially with UV light from the 
right ( $2^{\text {nd }}$ image) and from the left ( $4^{\text {th }}$ image), leading to a recovery of the initial form; b) $(i)$ Evolution of the volume change of an hydrogel consisting of a covalent polymer network similar to panel a) (i) but with stilbene units instead of azobenzene ones for different PEG sizes, upon sequential UV-A $(350 \mathrm{~nm})$ and UV-C $(280 \mathrm{~nm})$ irradiation, (ii) Tensile tests on these hydrogels made from different PEG sizes showing the influence of UV-A irradiation on the Young's modulus, (iii) Evolution of the flexion angle of a xerogel upon sequential UV-A and UV-C irradiation. Adapted with permissions from references 123 and 124.
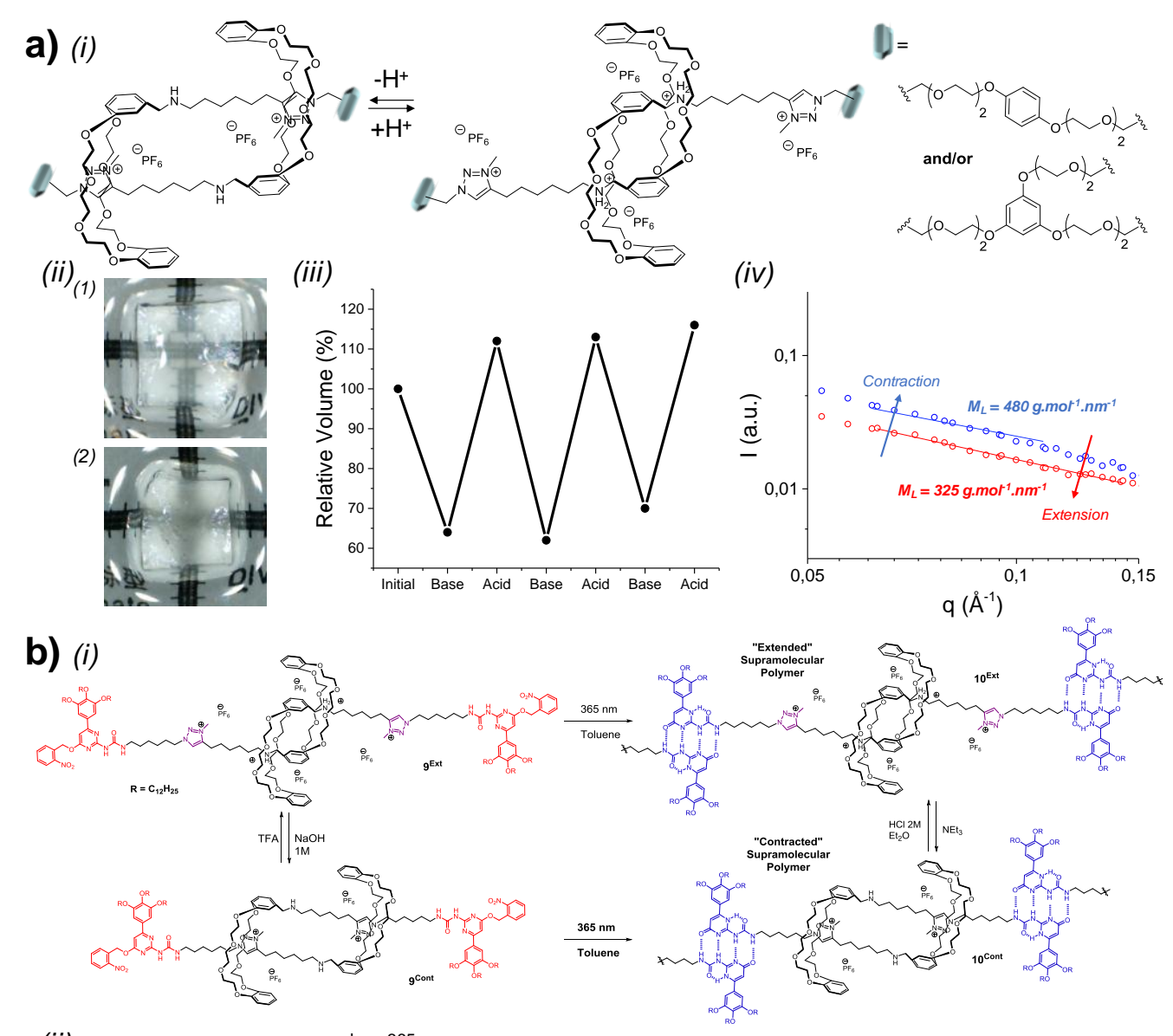

(ii)

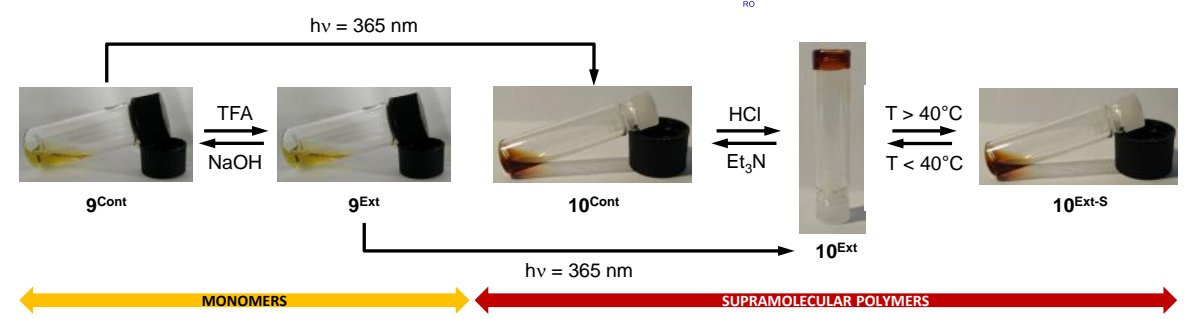

Figure 9. a) (i) Chemical structure of the covalent polymer network made of $\mathrm{pH}$-sensistive [c2]daisy chain rotaxanes, (ii) Corresponding chemically cross-linked gel (1) in its initial state and (2) after soaking in a basic solution for 15 min leading to a volume change from $100 \%$ to $63 \%$, (iii) Evolution of the relative volume of the chemical gel after three base/acid cycling events, (iv) Evolution of the neutron scattering curves recorded on the extended and contracted chemical gels highlighting the local actuation event (increased linear mass density for the contracted polymer (blue) vs. the extended one (red)); b) (i) Chemical structures of the $\mathrm{pH}$-responsive [ $c 2]$ daisy chain rotaxanes decorated with protected ureidopyrimidinone moieties (red) and their polymerization in toluene upon UV light irradiation, (ii) Images of the monomers and polymers showing a reversible gel-sol transition of the extended polymer 
using either $\mathrm{pH}$ or temperature as external triggers. Adapted with permissions from references 125 and 126.
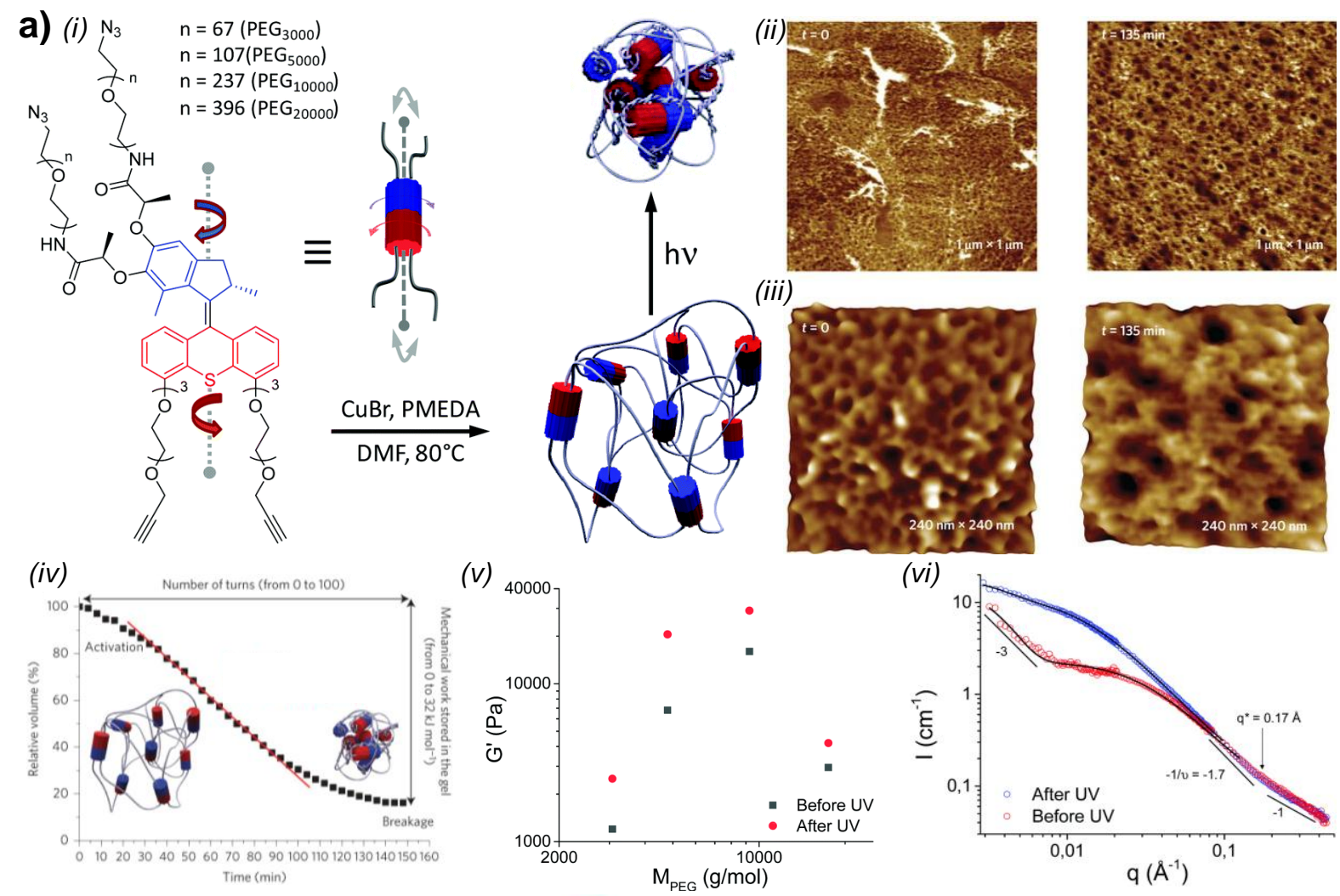

b) (i)
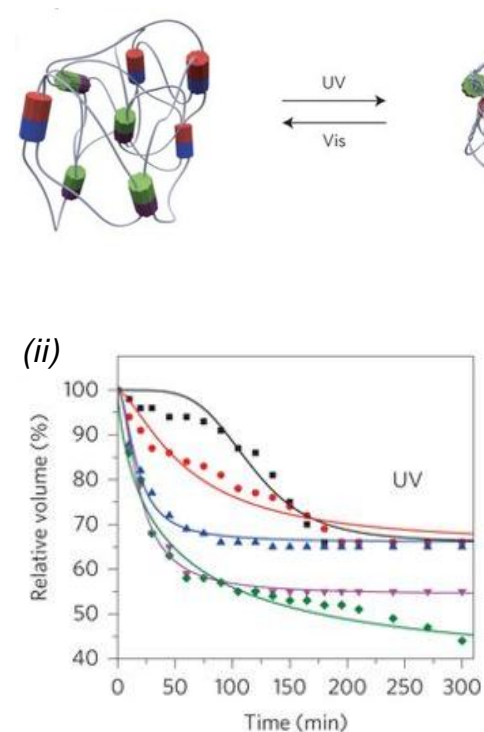
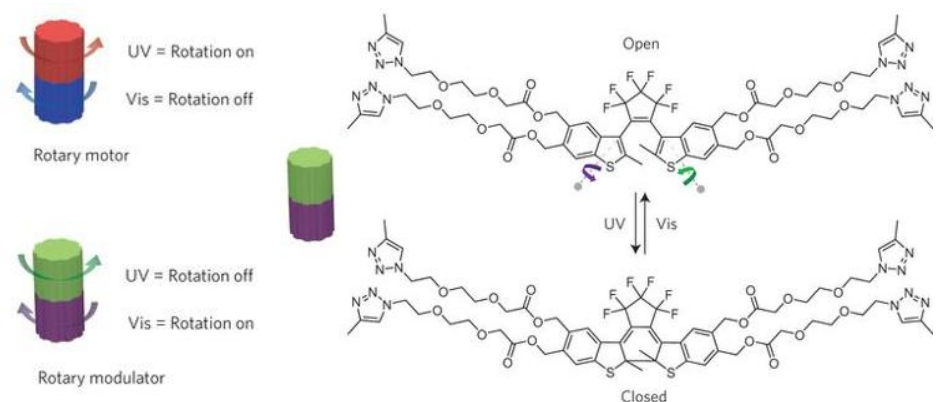

(iii)

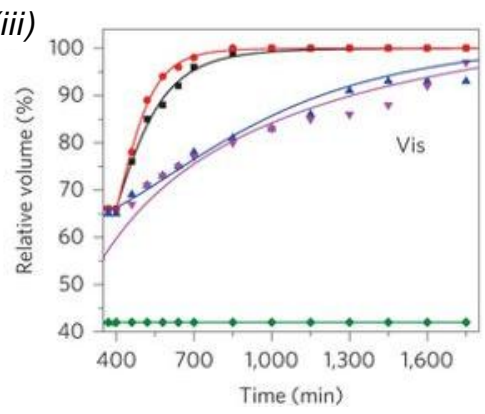

(iv)

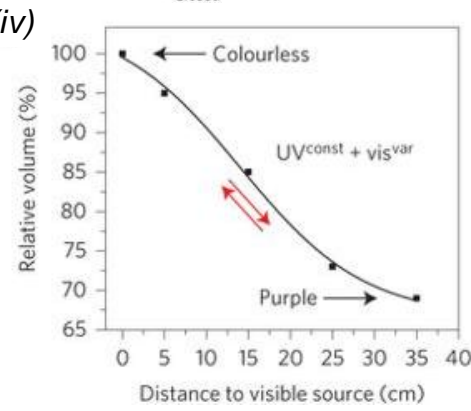

(vi)

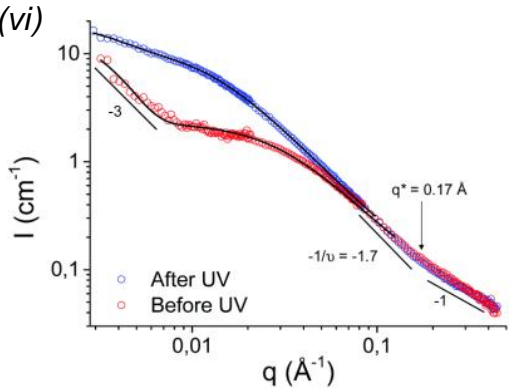

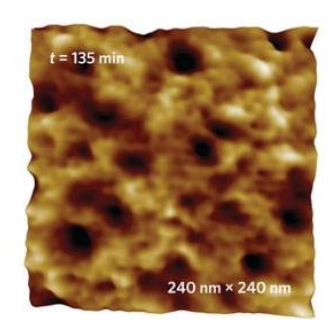

Figure 10. a) (i) Chemical structure of the motor-polymer conjugate with varying PEG sizes, its polymerization in a covalent polymer network under click chemistry conditions and schematic representation of its contraction upon UV light irradiation, (ii-iii) AFM images of a piece of gel containing rotary motors before irradiation (left images) and after 135 min of UV light irradiation (right images), (iv) Evolution of the relative volume of a piece of gel upon UV light irradiation over 160 minutes, (v) Evolution of the shear modulus G' of the chemical gel for different PEG sizes before and after UV light irradiation, (vi) Evolution of the small angle neutron scattering curves upon UV light irradiation for a gel prepared at $5 \mathrm{mM}$ and 
containing $5000 \mathrm{~g} / \mathrm{mol}$ PEG chains; b) (i) Schematic representation of a dual-light responsive chemical gel incorporating rotary motors and rotary modulators, chemical structures of the rotary modulator in its opened and closed forms, (ii-iii) Evolution of the relative volume of the corresponding gels from decreasing amounts of modulators (black: 50\%, red: $25 \%$, blue: $12 \%$, purple: $5 \%$ of modulators, green: no modulator) upon UV irradiation (ii) and subsequent visible light irradiation (iii), (iv) Evolution of the relative volume of a gel containing $50 \%$ of modulators upon simultaneous constant UV light irradiation and variable visible light irradiation. Adapted with permissions from references 128, 129 and 130.

\section{Biographies}

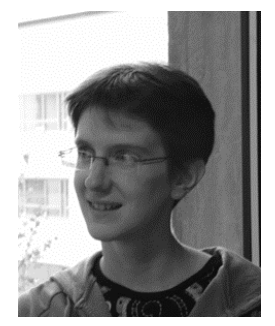

Emilie Moulin is a «Chargé de Recherche » CNRS at the Institut Charles Sadron in Strasbourg working in the group of Prof. Nicolas Giuseppone since 2008. In 2014, she completed her habilitation dedicated to the synthesis and characterization of complex supramolecular systems for materials science. Her research interests currently focus on combining polymers, supramolecular and organic chemistry to develop new functional materials.

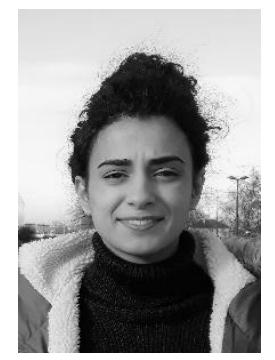

Lara Faour obtained her master's degree from the University of Angers in 2015. Later, she received her PhD degree in organic chemistry from the same university in 2018 working on aromatic foldamers under the supervision of Prof. Marc Sallé and Dr. David Canevet. Since, she joined the group of Prof. Nicolas Giuseppone as a postdoctoral researcher. Her research interests mainly concern supramolecular systems and functional materials.

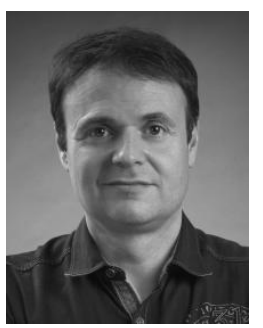

Nicolas Giuseppone is distinguished professor of chemistry (PRCE) at the University of Strasbourg, deputy director of the Institut Charles Sadron - CNRS, director of the Research Federation on Materials and Nanosciences - CNRS, and honorary member of the Institut Universitaire de France (IUF). His research interests are focused on supramolecular chemistry, molecular machines, and functional materials. 
New types of 2D and 3D stimuli-responsive materials can be constructed by integrating molecular machines as part of their building blocks. The transfer of mechanical actuations from the nanometric scale to the macroscopic one can be supported by several transmission processes, and offers a potential access to active functional materials for applications in medicine, information technologies, and robotics.

\section{Molecular Machines}

E. Moulin, L. Faour, C. C. Carmona-Vargas, and N. Giuseppone*

From molecular machines to stimuli-responsive materials

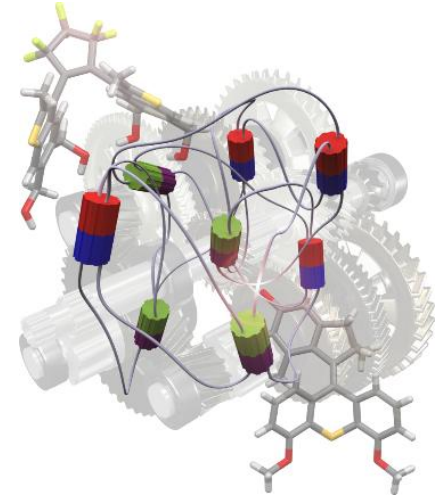

